Research Article

\title{
Mechanism of Radix Rhei Et Rhizome Intervention in Cerebral Infarction: A Research Based on Chemoinformatics and Systematic Pharmacology
}

\author{
Wang Xiang, ${ }^{1}$ Zhiyong Long, ${ }^{2}$ Jinsong Zeng $\left(\mathbb{D},{ }^{3,4}\right.$ Xiaofei Zhu, ${ }^{4}$ Mengxia Yuan, ${ }^{2}$ Jiamin Wu, \\ Yonghe $\mathrm{Wu},{ }^{4}$ and Liang $\mathrm{Liu}^{4}$ \\ ${ }^{1}$ The Affiliated Hospital of Guilin Medical University, Guilin, Guangxi Province, China \\ ${ }^{2}$ Shantou University Medical College, Shantou University, Shantou, Guangdong, China \\ ${ }^{3}$ The First Affiliated Hospital of Hunan University of Chinese Medicine, Changsha, Hunan Province, China \\ ${ }^{4}$ Hunan University of Chinese Medicine, Changsha, Hunan Province, China \\ ${ }^{5}$ Shanghai University of Traditional Chinese Medicine, Shanghai, China
}

Correspondence should be addressed to Jinsong Zeng; zengjingsong321@outlook.com

Received 31 May 2021; Accepted 13 August 2021; Published 7 September 2021

Academic Editor: Rajeev K Singla

Copyright (c) 2021 Wang Xiang et al. This is an open access article distributed under the Creative Commons Attribution License, which permits unrestricted use, distribution, and reproduction in any medium, provided the original work is properly cited.

Objective. To explore the therapeutic targets, network modules, and coexpressed genes of Radix Rhei Et Rhizome intervention in cerebral infarction (CI), and to predict significant biological processes and pathways through network pharmacology. To explore the differential proteins of Radix Rhei Et Rhizome intervention in CI, conduct bioinformatics verification, and initially explain the possible therapeutic mechanism of Radix Rhei Et Rhizome intervention in CI through proteomics. Methods. The TCM database was used to predict the potential compounds of Radix Rhei Et Rhizome, and the PharmMapper was used to predict its potential targets. GeneCards and OMIM were used to search for CI-related genes. Cytoscape was used to construct a protein-protein interaction (PPI) network and to screen out core genes and detection network modules. Then, DAVID and Metascape were used for enrichment analysis. After that, in-depth analysis of the proteomics data was carried out to further explore the mechanism of Radix Rhei Et Rhizome intervention in CI. Results. (1) A total of 14 Radix Rhei Et Rhizome potential components and 425 potential targets were obtained. The core components include sennoside A, palmidin A, emodin, toralactone, and so on. The potential targets were combined with $297 \mathrm{CI}$ genes to construct a PPI network. The targets shared by Radix Rhei Et Rhizome and CI include ALB, AKT1, MMP9, IGF1, CASP3, etc. The biological processes that Radix Rhei Et Rhizome may treat CI include platelet degranulation, cell migration, fibrinolysis, platelet activation, hypoxia, angiogenesis, endothelial cell apoptosis, coagulation, and neuronal apoptosis. The signaling pathways include Ras, PI3K-Akt, TNF, FoxO, HIF-1, and Rap1 signaling pathways. (2) Proteomics shows that the top 20 proteins in the differential protein PPI network were Syp, Syn1, Mbp, Gap43, Aif1, Camk2a, Syt1, Calm1, Calb1, Nsf, Nefl, Hspa5, Nefh, Ncam1, Dcx, Unc13a, Mapk1, Syt2, Dnm1, and Cltc. Differential protein enrichment results show that these proteins may be related to synaptic vesicle cycle, vesiclemediated transport in synapse, presynaptic endocytosis, synaptic vesicle endocytosis, axon guidance, calcium signaling pathway, and so on. Conclusion. This study combined network pharmacology and proteomics to explore the main material basis of Radix Rhei Et Rhizome for the treatment of CI such as sennoside A, palmidin A, emodin, and toralactone. The mechanism may be related to the regulation of biological processes (such as synaptic vesicle cycle, vesicle-mediated transport in synapse, presynaptic endocytosis, and synaptic vesicle endocytosis) and signaling pathways (such as Ras, PI3K-Akt, TNF, FoxO, HIF-1, Rap1, and axon guidance). 


\section{Introduction}

Cerebral infarction (CI) or ischemic stroke (IS) mainly results from blood supply disturbances in local brain tissue areas, leading to necrosis of ischemic hypoxic lesions in the brain tissue, which results in the manifestation of corresponding neurological deficits [1]. Epidemiological studies have shown that stroke has become the disease with the highest mortality rate in China $[2,3]$. CI is divided into cerebral thrombosis, cerebral embolism, and lacunar infarction according to the different pathogenesis. Among them, cerebral thrombosis is the most common type of CI, accounting for about $60 \%$ [4]. Timely thrombolysis to restore blood supply after infarction is the most important measure to save the ischemic area. Although reperfusion after ischemia can restore its function, ischemia-reperfusion injury makes the irreversible damage to the brain tissue after the blood flow restored [5-7]. Cerebral ischemia-reperfusion injury (CIR) is mainly related to the formation of free radicals (oxygen and lipid free radicals), oxidative stress, energy metabolism disorders, apoptosis, excitatory amino acid toxicity, calcium overload, inflammation, and so on [6-9]. Currently, the preventive and therapeutic drugs for CIR include excitatory amino acid-regulating drugs, neurotrophic growth factors, free radical scavengers, nitric oxide synthase inhibitors, intracellular calcium overload inhibitors, and natural plant active compounds (flavonoids, saponins, polysaccharides) $[8,10-13]$. Of particular importance is that natural plant active compounds are becoming potential CIR drugs.

Radix Rhei Et Rhizome is an important part of the traditional Chinese medicine (TCM) formulas for the treatment of CI in the acute phase, which has a long history of medicinal use [14-18]. Modern medical research proves that rhubarb aglycones have significant protective effects on ischemic brain tissue: it can maintain the integrity of the blood-brain barrier, reduce inflammation, inhibit apoptosis, and protect nerves [15-19]. However, its specific mechanism is still unclear. Therefore, this research hopes to propose a new method to analyze the regulatory mechanism of Radix Rhei Et Rhizome on CI biological networks. The development of high-throughput omics and chemoinformatics has given the opportunity to analyze the mechanisms of natural plant components for disease treatment [20-24]. Therefore, based on previous research, this study will integrate proteomics and chemoinformatics strategies to further explore the molecular mechanism of Radix Rhei Et Rhizome's intervention in CI and provide reference information for new drug development and its clinical application. The idea and process of this research are shown in Figure 1.

\section{Material and Methods}

2.1. Construction of Pharmacodynamic Molecular Database and Radix Rhei Et Rhizome's Compounds Prediction. All compounds of Radix Rhei Et Rhizome were obtained from the traditional Chinese medicine database and analysis platforms TCMSP database (http://lsp.nwu.edu.cn/) [25] and TCM@Taiwan (http://tcm.cmu.edu.tw/zh-tw/) [26]. In order to obtain potential active compounds from these compounds, this study used drug-likeness (DL), Caco-2 permeability, and oral bioavailability (OB) indicators [20-24, 27-30] and combined literature [31] to predict potential pharmacological compounds in Radix Rhei Et Rhizome. The standard was $\mathrm{OB} \geq 30 \%, \mathrm{DL} \geq 0.18$, and Caco2 permeability $>-0.4$. After the potential compound prediction, a total of 9 Radix Rhei Et Rhizome's potential compounds were obtained: (-)-catechin, aloe-emodin, betasitosterol, daucosterol, eupatin, mutatochrome, palmidin A, rhein, and toralactone. Meanwhile, due to the limitation of the pharmacokinetic parameter model, in order to avoid the omission of potential compounds, a large number of studies related to Radix Rhei Et Rhizome were searched to supplement its active compounds. Finally, according to references $[32,33]$, a total of 5 oral absorbable compounds with bioactivity were supplemented: chrysophanol, danthron, emodin, sennoside $\mathrm{A}$, and physcion. The $3 \mathrm{D}$ structure of all screened compounds was saved in mol2 format.

\subsection{Potential Targets Prediction and CI Gene Collection.} In addition to screening the active components of Radix Rhei Et Rhizome, determining the targets of the active ingredients is also an important step to clarify the biological basis of TCM. The PharmMapper server platform (http://lilab-ecust. $\mathrm{cn} /$ pharmmapper/) was used to predict potential targets. After importing the "mol2" format file, the number of returned targets was set to 300 , and the pharmacophore model was selected as the setting condition [34]. The PDB ID of the protein target was imported into UniProt KB (https:// www.uniprot.org/uniprot/), with the species restricted to "Homo sapiens" (for potential targets) (Table S1) or "Rattus norvegicus" (for proteomics data) (Table S2), to obtain the official symbol of Radix Rhei Et Rhizome potential target.

The keyword "cerebral infarction" was entered into the GeneCards database (http://www.genecards.org/) [35] and the OMIM database (http://www.ncbi.nlm.nih.gov/omim) [36] to search for reported CI-related genes. The genes in the GeneCards database with relevance score $>1$ were selected. After removing duplicate genes and false positive genes, the CI gene set was obtained (Table S3).

2.3. Network Construction and Analysis Methods. In system pharmacology, the construction and analysis of biological network diagrams are very important for TCM pharmacological analysis. The network formed by nodes and edges (connections between nodes) is a mathematical-based and quantifiable mapping of various regulatory relationships under complex biological systems. String 11.0 (https://string-db.org/) was used to query protein-protein interaction (PPI) relationships [37]. The results were saved in TSV format, and the node1, node2, and Combinedscore information in the file was retained and imported into Cytoscape 3.7.1 software to draw the relevant network [38]. The "NetworkAnalyzer" plugin that comes with Cytoscape software was used to analyze the degree and betweenness of the network. These two parameters are often used to illustrate the importance of nodes, that is, the higher the degree and betweenness, the more important the 


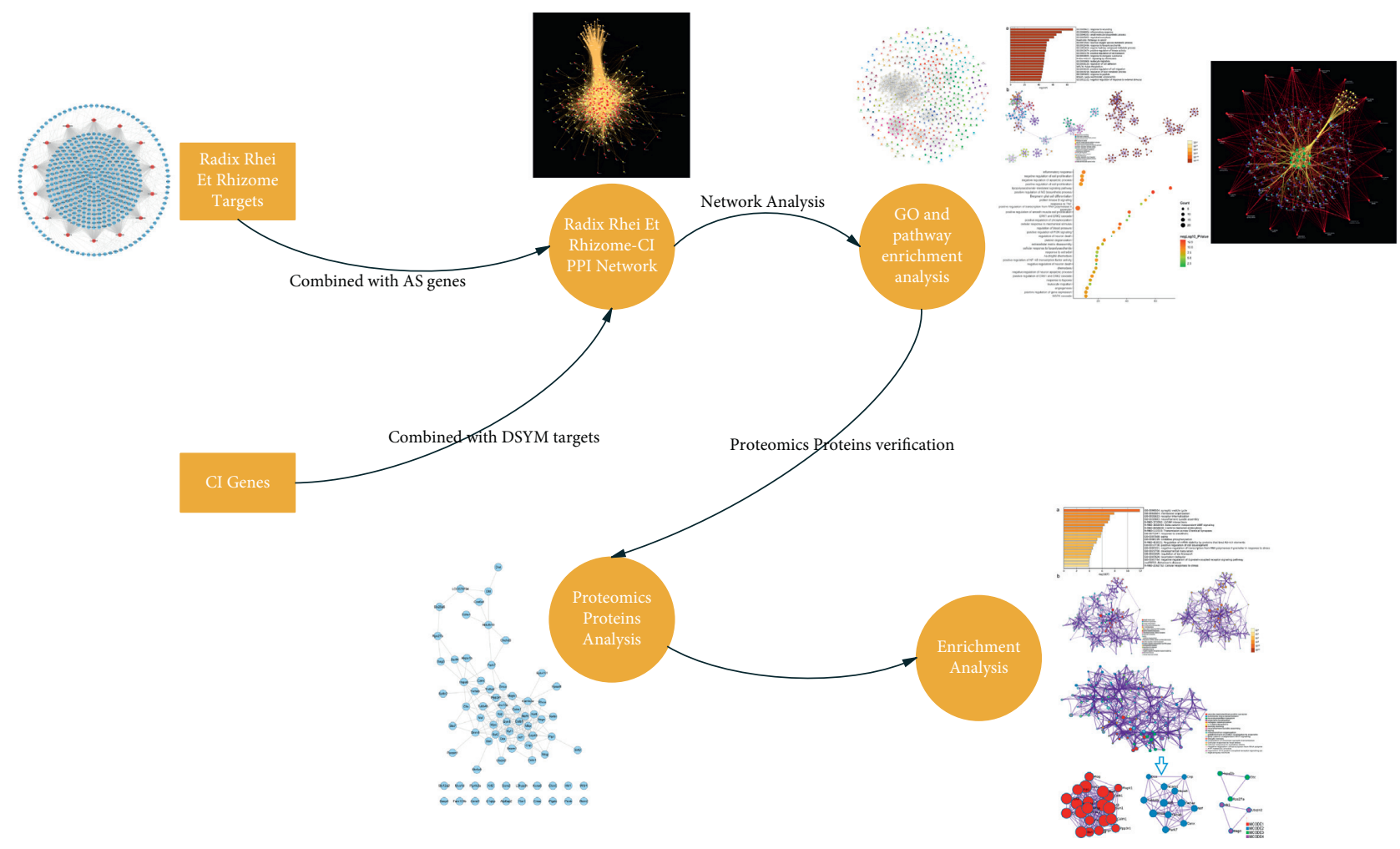

Figure 1: The idea and process of this research.

node in the network. The clusters of networks were detected by MCODE (Cytoscape's plugin). The MCODE algorithm was originally a clustering algorithm designed to detect protein complexes in PPI networks, which can detect tightly connected regions (i.e., molecular complexes) in large-scale protein interaction networks [38]. This method can now also be used to detect clusters in other types of networks.

2.4. Gene Ontology (GO) Enrichment, Pathway Enrichment, and Reactome Enrichment Analysis. DAVID ver. 6.8 (https:// david-d.ncifcrf.gov) was used for the GO enrichment analysis of targets and genes in clusters and for the pathway enrichment analysis of targets and genes in PPI networks [39]. The Reactome Pathway Database (https://reactome.org/) was used for reactome pathway enrichment [40].

\section{Results and Discussion}

3.1. Potential Compound-Potential Target Network of Radix Rhei Et Rhizome. A total of 14 components and 425 targets were used to construct the potential compound-potential target network of Radix Rhei Et Rhizome. In this network, nodes near the center have a greater degree than nodes near the periphery (Figure 2).

\subsection{Radix Rhei Et Rhizome-CI PPI Network Analysis}

3.2.1. Radix Rhei Et Rhizome-CI PPI Network Construction. The Radix Rhei Et Rhizome-CI PPI network is composed of 645 nodes (371 potential target nodes, 231 CI gene nodes, and 43 Radix Rhei Et Rhizome-CI target nodes) and 14,119 edges. The following are the top 20 nodes in the network: (1) Radix Rhei Et Rhizome targets: EGFR (203 edges), SRC (201 edges), MAPK1 (193 edges), and MAPK8 (167 edges). (2) CI genes: INS (292 edges), IL6 (273 edges), VEGFA (244 edges), TNF (241 edges), TP53 (235 edges), EGF (210 edges), CXCL8 (178 edges), IL10 (163 edges), IL1B (160 edges), CCL2 (159 edges), and APP (157 edges). (3) Radix Rhei Et Rhizome-CI targets: ALB (302 edges), AKT1 (266 edges), MMP9 (191 edges), IGF1 (182 edges), and CASP3 (170 edges) (Figure 3 ). The preliminary enrichment results of biological processes and signaling pathways are shown in Figures 4 and 5 .

In this study, a total of 14 Radix Rhei Et Rhizome compounds and 425 potential targets were predicted for analysis using the network pharmacological method. Although the number of predicted targets for each potential compound is different, the overlap of the target set of some compounds is large. In other words, Radix Rhei Et Rhizome's compounds have common targets probably because these compounds come from the same structural parent. For example, rhein, aloe-emodin, chrysophanol, physcion, and emodin are known as rhubarb aglycones.

In terms of the blood-brain barrier, studies have shown that emodin can maintain the integrity of the blood-brain barrier, reduce inflammation, and inhibit apoptosis [41-45]. In another study, emodin reduced blood-brain barrier permeability and reduced infarct size by inhibiting the expression of connexin $43(\mathrm{Cx} 43)$ and aquaporin 4 (AQP4) in cerebral ischemia/reperfusion model rats [46]. In terms of inhibiting inflammation, 


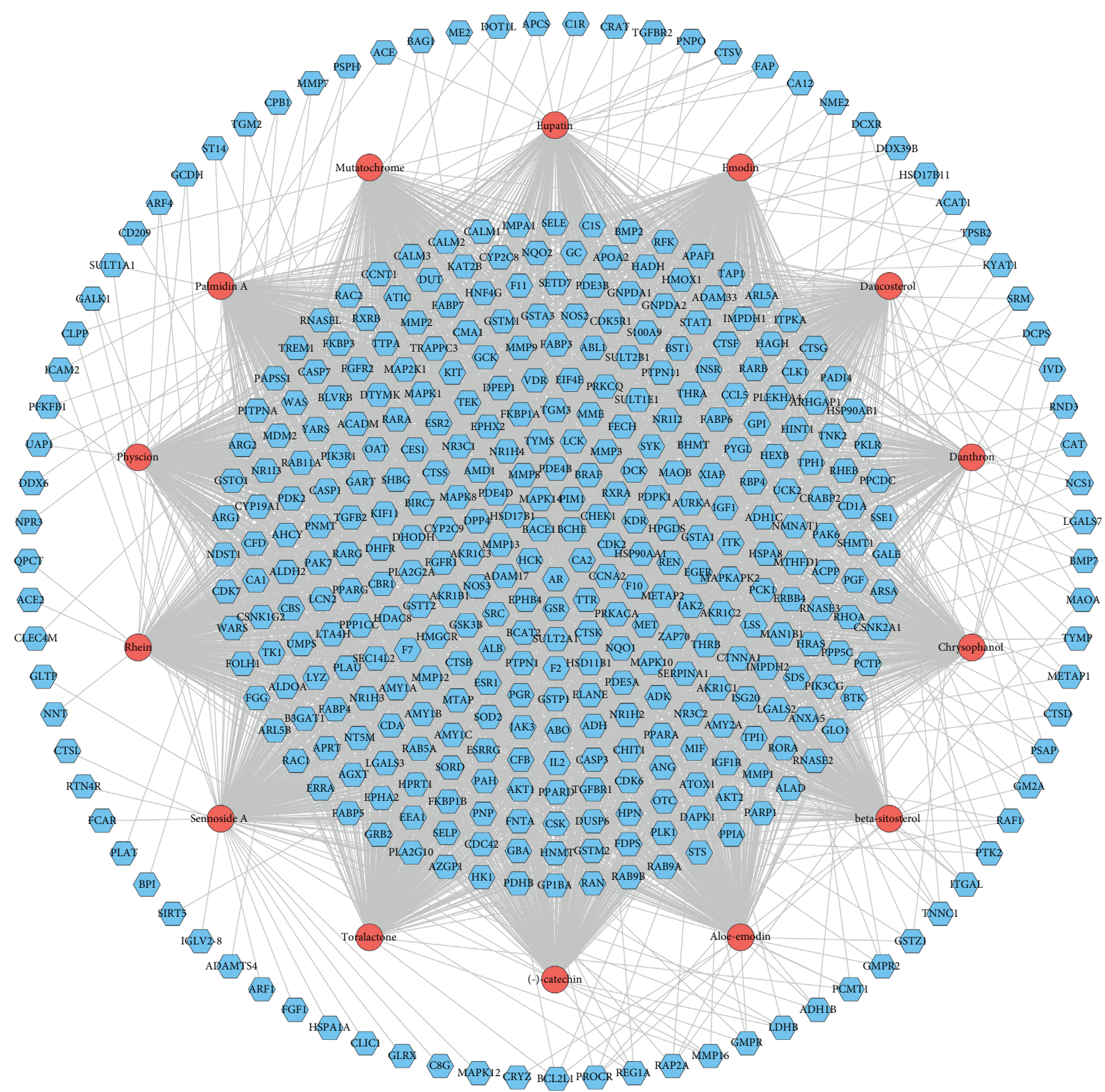

Figure 2: Potential Compound-Potential Target Network of Radix Rhei Et Rhizome (Blue hexagons stand for potential targets. Red circles stand for potential compounds.).

emodin can inhibit transforming growth factor (TGF)- $\beta$, tumor necrosis factor (TNF)- $\alpha$, interleukin (IL)- $1 \beta$, and intercellular adhesion molecule 1 (ICAM-1), so as to protect the brain [42]. Chrysophanol inhibits the inflammatory response by reducing the expression of IL- $1 \beta$, caspase-1, and NALP3, thereby improving neurological deficits, infarct volume, cerebral edema, and blood-brain barrier permeability in mice with ischemia-reperfusion injury. Chrysophanol can also improve the survival rate, nervous system score, and motor function of mice with middle cerebral artery occlusion by reducing the expression of TNF- $\alpha$, IL- $1 \beta$, and NF- $\kappa$ B p65 [47]. In terms of inhibiting apoptosis, emodin can inhibit neuronal apoptosis $[43,44,48]$. Its specific mechanism may be that emodin can increase Bcl-2 and inhibit caspase- 3 and Bax expression to reduce glutamate-induced HT22 cell apoptosis [43]. Rhein increases the expression of mature brainderived neurotrophic factor (BDNF) and phosphorylation of Akt and cAMP response element binding protein (CREB), which improves the behavior and function of CI mice [49]. Rhein also reduced the expression of BAX, caspase- 9 , caspase- 3 , and cleaved caspase- 3 and increased the expression of $\mathrm{Bcl}-2$, thereby reducing the infarcted area of cerebral ischemia-reperfusion injury mice [50]. In addition, chrysophanol can inhibit NO-related neuronal cell death by attenuating nitrite and nitrate (NOx-) and 3nitrotyrosine (3-NT) levels and reducing lysed caspase-3 protein expression [51]. 


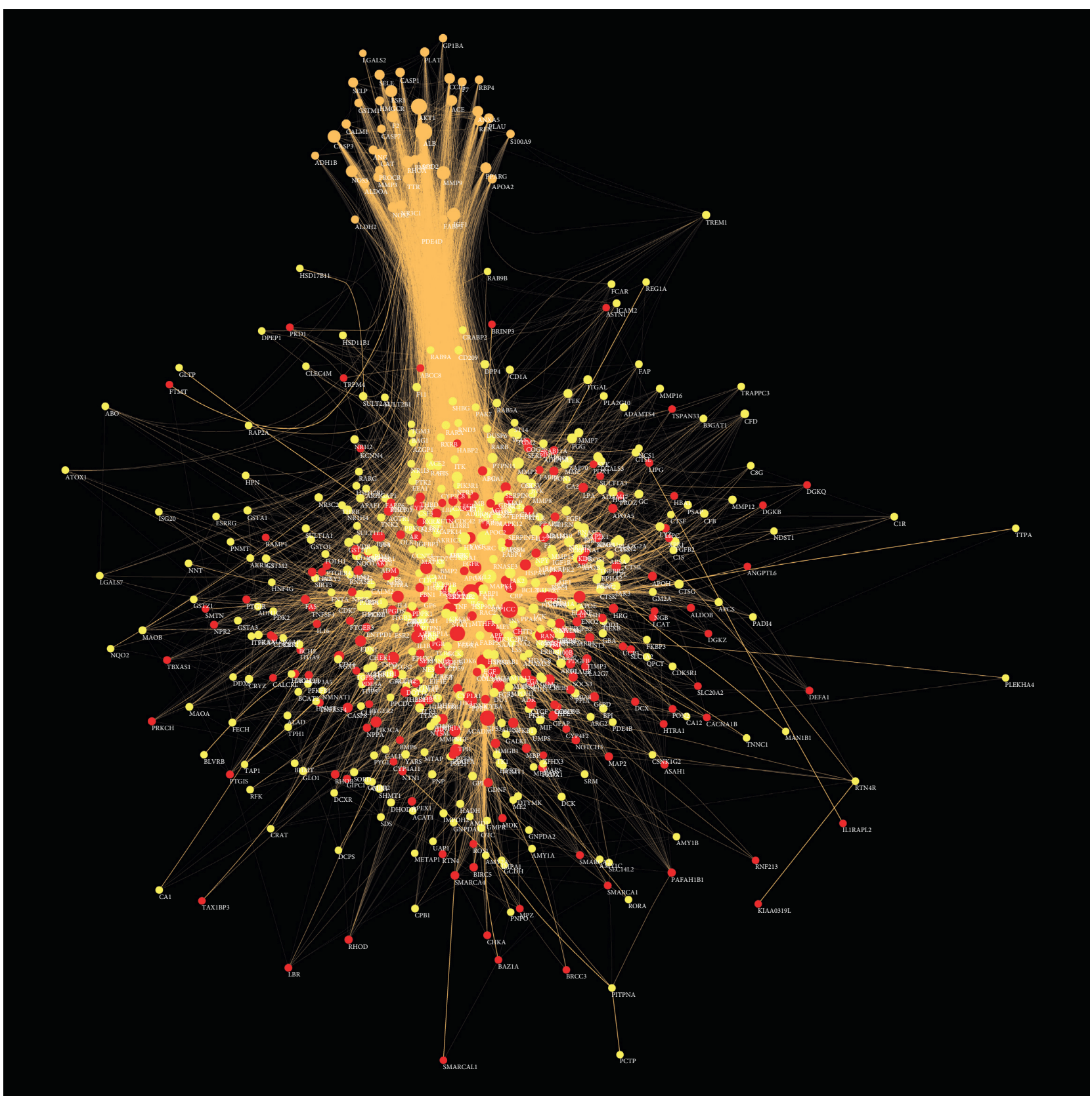

Figure 3: Radix Rhei Et Rhizome-CI PPI network (Red, yellow, and orange circles stand for CI genes, Radix Rhei Et Rhizome targets, and Radix Rhei Et Rhizome-CI targets, respectively. The larger the node size, the higher the degree of the node. The thicker the line, the greater the edge betweenness of the node.).

In terms of oxidative stress, emodin inhibits the apoptosis of primary rat cortical neurons induced by hydrogen peroxide $\left(\mathrm{H}_{2} \mathrm{O}_{2}\right)$ [44]. It was also found that emodin can inhibit the apoptosis of neurons after oxyglucose deprivation and reduce the damage of PC12 nerve cells by increasing the expression of activin A [45]. Chrysophanol also increases total superoxide dismutase (SOD) and manganese-dependent SOD (MnSOD) activities in cerebral ischemia-reperfusion injury models and inhibits the production of reactive oxygen species (ROS) [51]. In addition, rhein can reduce malondialdehyde (MDA) and increase the activities of SOD, catalase (CAT), and glutathione peroxidase (GSH$\mathrm{Px})$ and improve neurological function scores [50].
Chrysophanol can improve endoplasmic reticulum (ER) stress by reducing ER stress-related factors (such as glucose-regulated protein 78 (GRP78), phosphorylated eukaryotic initiation factor $2 \alpha$ (p-eIF $2 \alpha)$, CCAAT-enhancer-binding protein homologous protein (CHOP), caspase-12, and NF- $\kappa \mathrm{B} / \kappa \mathrm{B}-\alpha)$ [52].

In terms of various compound combinations and synergies, rhubarb aglycones (aloe-emodin, rhein, emodin, chrysophanol, and physcion) can improve disorders of amino acid, energy, and lipid metabolism caused by cerebral ischemia-reperfusion injury [15]. Further research shows that rhubarb aglycones can reduce IgG content and increase type IV collagen (CoLIV) and laminin (LN) levels, thereby 


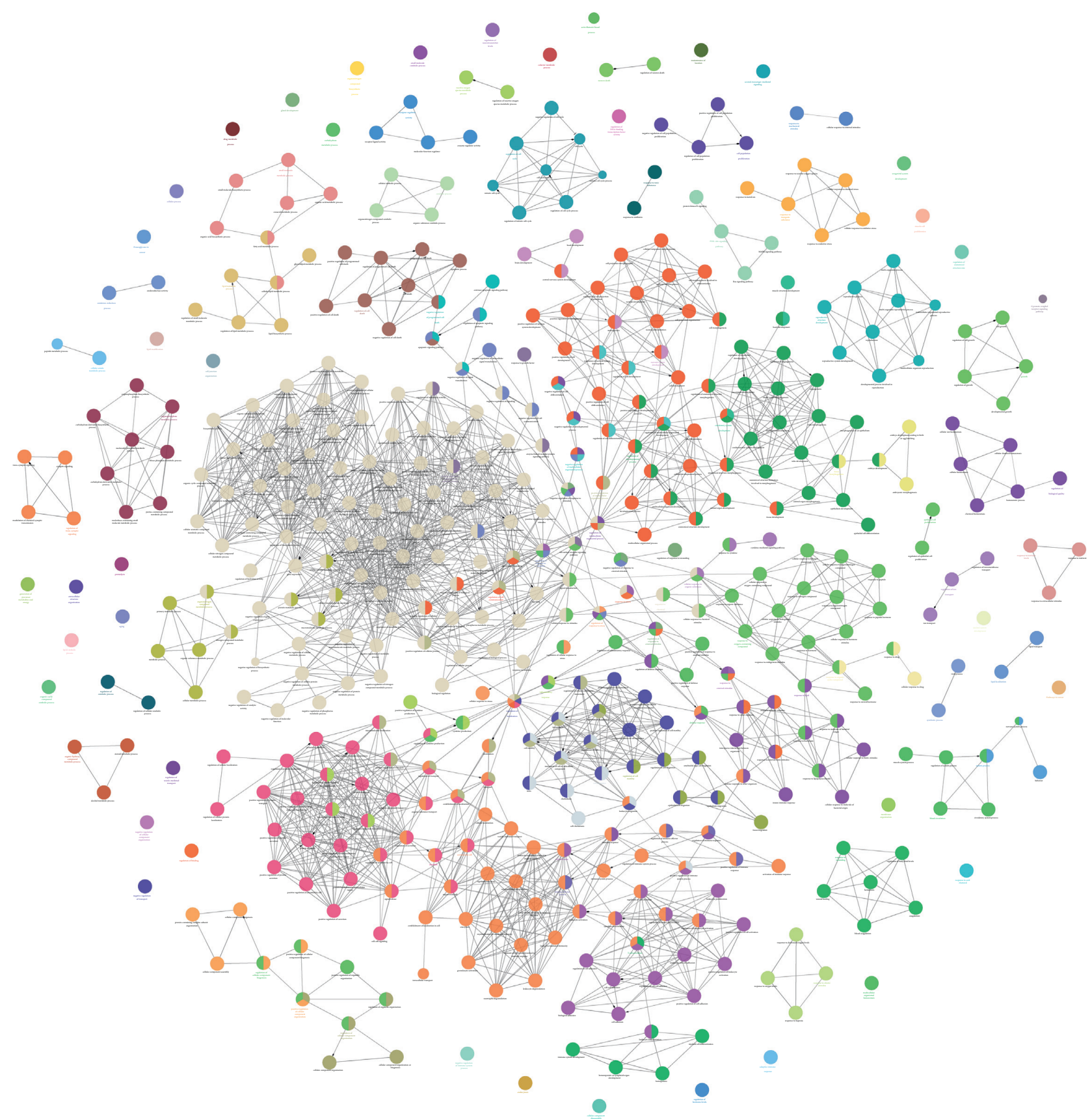

FIgURE 4: The preliminary enrichment results by ClueGO.

reducing cerebral microvascular basement membrane damage caused by thrombolysis [53]. Pharmacokinetic studies have shown that in CI model rats, the maximum plasma concentration ( $\mathrm{C} \max$ ), half-life ( $\mathrm{t} 1 / 2)$, and area under the curve (AUC $0-t$ ) increased significantly, but the overall clearance (CL) value decreased significantly, indicating that rhubarb anthraquinones are more easily absorbed after coadministration [54].

3.2.2. Biological Processes of Radix Rhei Et Rhizome-CI PPI Network. Eighteen (18) clusters returned after analyzed by MCODE (Table 1 and Figure 6). The cluster score (complex score) is defined as the product of the complex subgraph, $C=(V, E)$, density, and the number of vertices in the complex subgraph $(\mathrm{DC} \times|\mathrm{V}|)$. The higher the score, the denser the cluster.

The potential targets and CI genes in the cluster were introduced into DAVID for GO enrichment analysis. The biological process of the top 10 clusters is taken as an example (Table S4). For example, cluster 1 is related to GO: 0045429, GO:0031663, GO:0048661, GO:0071260, GO: 0045944, GO:0008217, GO:0006954, GO:0043066, GO: 0002576, GO:0070374, and GO:0051092; cluster 2 is associated with GO:0002576, GO:0070374, GO:0043066, GO: 0030335, GO:0001934, GO:0010628, GO:0042730, GO: 


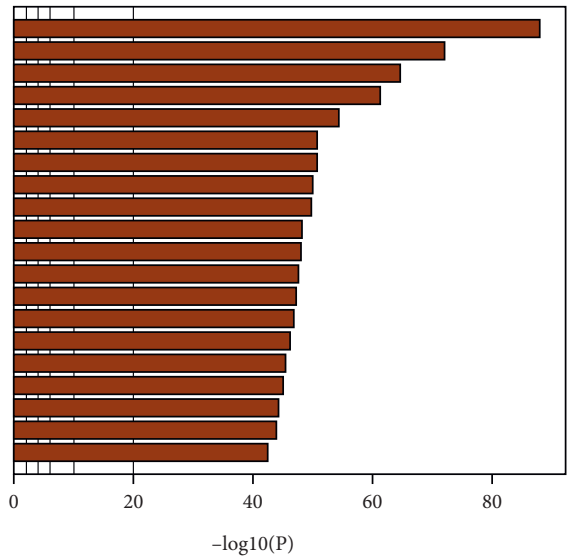

GO:0009611: response to wounding

GO:0006954: inflammatory response

GO:0044283: small molecule biosynthetic process

GO:0045055: regulated exocytosis

hsa05200: Pathways in cancer

GO:0072593 : reactive oxygen species metabolic proces

GO:0032496: response to lipopolysaccharide

GO:1901615: organic hydroxy compound metabolic process

GO:0033674: positive regulation of kinase activity

GO:0043270: positive regulation of ion transport

GO:0010035: response to inorganic substance

R-HSA-449147 : Signaling by lnterleukins

GO:0050900: leukocyte migration

GO:0030155: regulation of cell adhesion

WP176: Folate Metabolism

GO:0030335: positive regulation of cell migration

GO:0019216: regulation of lipid metabolic process

GO:1901652: response to peptide

M5885: NABA MATRISOME ASSOCIATED

GO:0032102: negative regulation of response to external stimulus

(a)
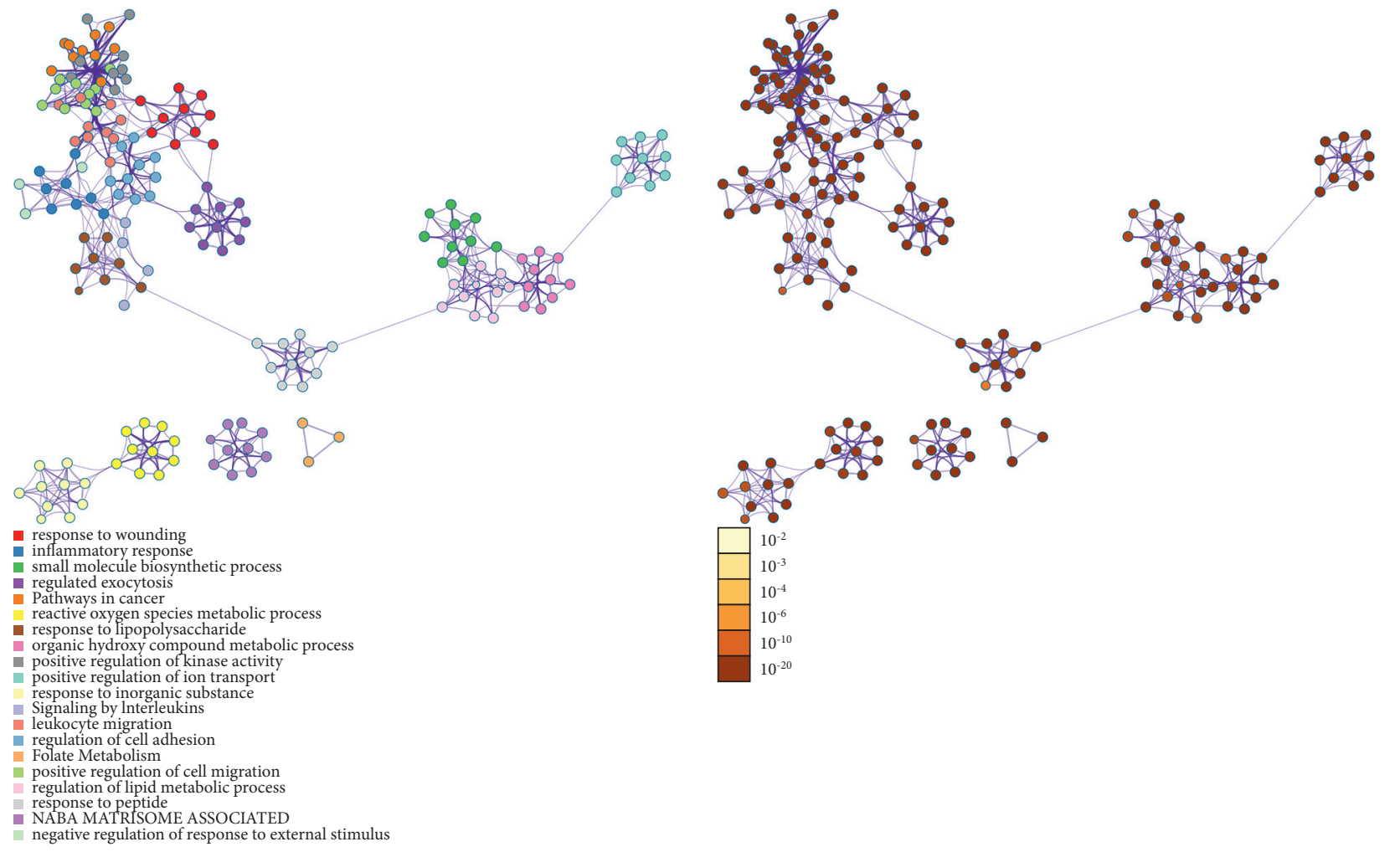

(b)

Figure 5: The Metascape results: (a) top biological processes, signaling pathways, and reactome pathways and (b) PPI network colored by enrichment results or $P$-values.

0030168, GO:0007165, GO:0043406, and GO:0043065; and cluster 3 is involved in GO:0042632, GO:0019433, GO: 0042157, GO:0017187, GO:0007584, GO:0034375, GO: 0043691, GO:0019915, GO:0006465, and GO:0070328. The details of clusters and biological processes are shown in Table S4. Since the biological processes in cluster 1 are representative, the main biological processes of cluster 1 are shown as an example (Figure 7).

3.2.3. Signaling Pathways of Radix Rhei Et Rhizome-CI PPI Network. Nineteen (19) CI-related signal pathways were returned. The relationship among signaling pathways, targets, and components is shown in Figure 8. The details of signaling pathways are shown in Figure 9 and Table S5. The number of targets regulated by the components of Radix Rhei Et Rhizome is shown in Table 2.

\subsubsection{Reactome Pathways of Radix Rhei Et Rhizome-CI PPI} Network. Ninety-three (93) CI-related signal pathways were returned. The relationship among reactome pathways, targets, and components is shown in Figure 10. The details of reactome pathways are shown in Figure 11 (Table S6). The number of targets regulated by the components of Radix Rhei Et Rhizome is shown in Table 3. 
TABle 1: Clusters of Radix Rhei Et Rhizome-CI PPI network.

\begin{tabular}{|c|c|c|c|c|}
\hline Cluster & core & Jodes & Edges & Targets and genes \\
\hline 1 & 44 & 67 & 1452 & $\begin{array}{l}\text { IGF1, NOS3, SERPINE1, MMP2, CRP, CCL5, VWF, PTEN, CASP8, MYD88, CD40, TLR4, MMP1, PLG, } \\
\text { NGF, CTGF, HRAS, HSPA4, HIF1A, MMP9, RHOA, ANXA5, CREB1, SOD2, REN, CYCS, GRB2, } \\
\text { MAPK1, MMP3, BCL2L1, IL2, TGFB1, ADIPOQ, AGT, FGF2, SELE, CD40LG, PTGS2, ALB, IL10, IL1B, } \\
\text { HGF, MAP2K1, CCL2, IL4, ACE, CAT, THBS1, CXCL8, PPARG, AR, JAK2, ESR1, HMOX1, MMP7, } \\
\text { CSF3, EDN1, BDNF, EGFR, SELP, MAPK14, CDC42, ICAM1, MMP13, STAT1, APOE, APP }\end{array}$ \\
\hline 2 & 25.821 & 79 & 1007 & $\begin{array}{l}\text { AKT1, XIAP, PIK3CA, SRC, F13A1, ALDOA, F3, NFKB1, AKT2, PTPN11, FGA, FGG, AGTR1, NR3C1, } \\
\text { AIF1, PPBP, HSP90AA1, SERPINF2, NQO1, NOS2, ABL1, FGB, SOCS3, CD34, MIF, BMP2, PDGFRB, } \\
\text { PDGFB, MET, HMGB1, IL6, KIT, PGF, MDM2, HPGDS, TEK, MBP, LGALS3, ENG, SOD1, MAPK8, } \\
\text { TNF, PLAU, GFAP, PTK2, INS, RAF1, IL1A, GSK3B, TP53, CASP3, FAS, PTPN1, PROS1, TGFB2, } \\
\text { VEGFA, HRG, ELANE, IGFBP3, TLR3, MAPK10, PGR, NCF1, CCNA2, CASP1, FGF1, IGF1R, F8, } \\
\text { PLAUR, RETN, ADAM17, LDLR, KDR, CFD, MMP14, F5, PARP1, S100B, EGF }\end{array}$ \\
\hline 3 & 6.846 & 27 & 89 & $\begin{array}{l}\text { PAH, LPL, ARSA, RNASE2, APCS, CTSK, IMPDH1, CTSL, PON1, FABP4, RBP4, LPA, BPI, CST3, } \\
\text { PROZ, RNASE3, SCARB1, F7, LIPG, FABP5, GM2A, PROC, HMGCR, GC, HABP2, HEXB, APOA1 } \\
\text { F11, SERPINC1, HP, APOM, AKR1B1, HK1, HSP90AB1, SERPINA1, APOB, ENO2, ABCA1, OLR1, }\end{array}$ \\
\hline 4 & 5.706 & 35 & 97 & $\begin{array}{c}\text { ATIC, PIK3CG, PLA2G7, CDK6, F10, ANG, SERPIND1, ZAP70, RHEB, LCAT, BACE1, HCK, MMP12, } \\
\text { BTK, TTR, COG2, LIPC, ITGAL, AURKA, SYK, BRAF, APOC2, F12 }\end{array}$ \\
\hline 5 & 4.783 & 24 & 55 & $\begin{array}{l}\text { PNP, BHMT, HADH, NR1I3, NT5M, SHMT1, YARS, TPI1, RAN, STS, UCK2, UCP3, ACADM, TYMS, } \\
\text { RXRA, AHCY, LDHB, IVD, CYP1A1, PDE3B, PSPH, ADK, SULT1A1, AKR1C3 }\end{array}$ \\
\hline 6 & 4 & 21 & 40 & $\begin{array}{l}\text { G6PD, MAP2, RAC2, GLRX, PTGER3, GSTP1, RHOB, RHOD, ASAH1, DCX, PSAP, CHIT1, CALM1, } \\
\text { CRYZ, CTSS, CDA, PRDX1, HTR1A, LTA4H, QPCT, NPY }\end{array}$ \\
\hline 7 & 4 & 8 & 14 & GLO1, GMPR, APRT, APEX1, IMPDH2, UMPS, DTYMK, TK1 \\
\hline 8 & 4 & 4 & 6 & RXRB, THRB, RARG, RARB \\
\hline 9 & 4 & 5 & 0 & GALE, GNPDA2, UAP1, GALK1, GNPDA1 \\
\hline 10 & 3.818 & 34 & 63 & $\begin{array}{c}\text { APOH, FBN1, TIMP3, THBD, HSPA8, EPHA2, INSR, PF4, ADAM10, PLAT, F2, RAC1, APAF1, } \\
\text { PIK3R1, ERBB4, GSR, CASP7, CDK2, CHEK1, NOS1, EIF4E, HSPA1A, ESR2, CCL11, CYBA, FGFR2, } \\
\text { ARG1, JAK3, APOA2, CSK, LCK, TGFBR1, MMP8, LCN2 }\end{array}$ \\
\hline 11 & 3.75 & 17 & 30 & $\begin{array}{l}\text { GSTO1, CYP2C8, AGXT, GSTA1, FCAR, PCK1, BST1, ADH1C, P2RY12, ITK, ITGB2, CYP3A5, } \\
\text { ADH1B, PYGL, NR1H4, HLA-DRB1, CYP2C9 }\end{array}$ \\
\hline 12 & 3.571 & 15 & 25 & $\begin{array}{c}\text { SLC9A1, FABP1, KAT2B, SAA1, APOA5, TBXA2R, PPARA, ACTA2, TGFBR2, PRKACA, CPB2, RARA, } \\
\text { EDNRA, COL3A1, NR1H3 }\end{array}$ \\
\hline 13 & 3.33 & 4 & $J$ & GSTM2, SOD3, GSTA3, GSTM1 \\
\hline 14 & 3 & 3 & 3 & GPI, PKLR, ALDOB \\
\hline 15 & 3 & 3 & 3 & SMARCA1, DOT1L, HDAC8 \\
\hline 16 & 3 & 3 & 3 & MAOA, PNMT, MAOB \\
\hline 17 & 3 & 3 & 3 & AMY1A, AMY1B, AMY1C \\
\hline 18 & 2.889 & 10 & 13 & GART, DCK, HFE, FECH, DHFR, PDE4B, ALAD, CLPP, PDE4D, DHODH \\
\hline
\end{tabular}

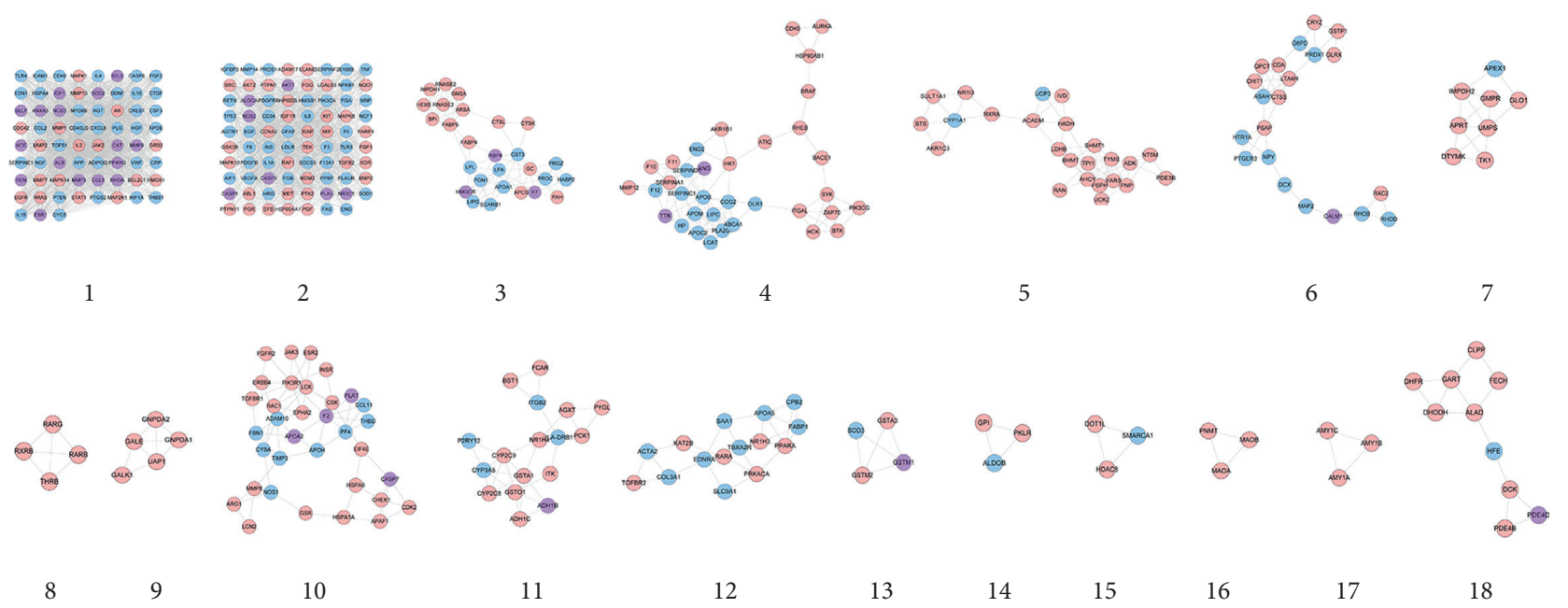

FIgURe 6: Clusters of Radix Rhei Et Rhizome-CI PPI network (Blue, pink, and purple circles stand for CI genes, Radix Rhei Et Rhizome targets, and Radix Rhei Et Rhizome-CI targets, respectively.). 


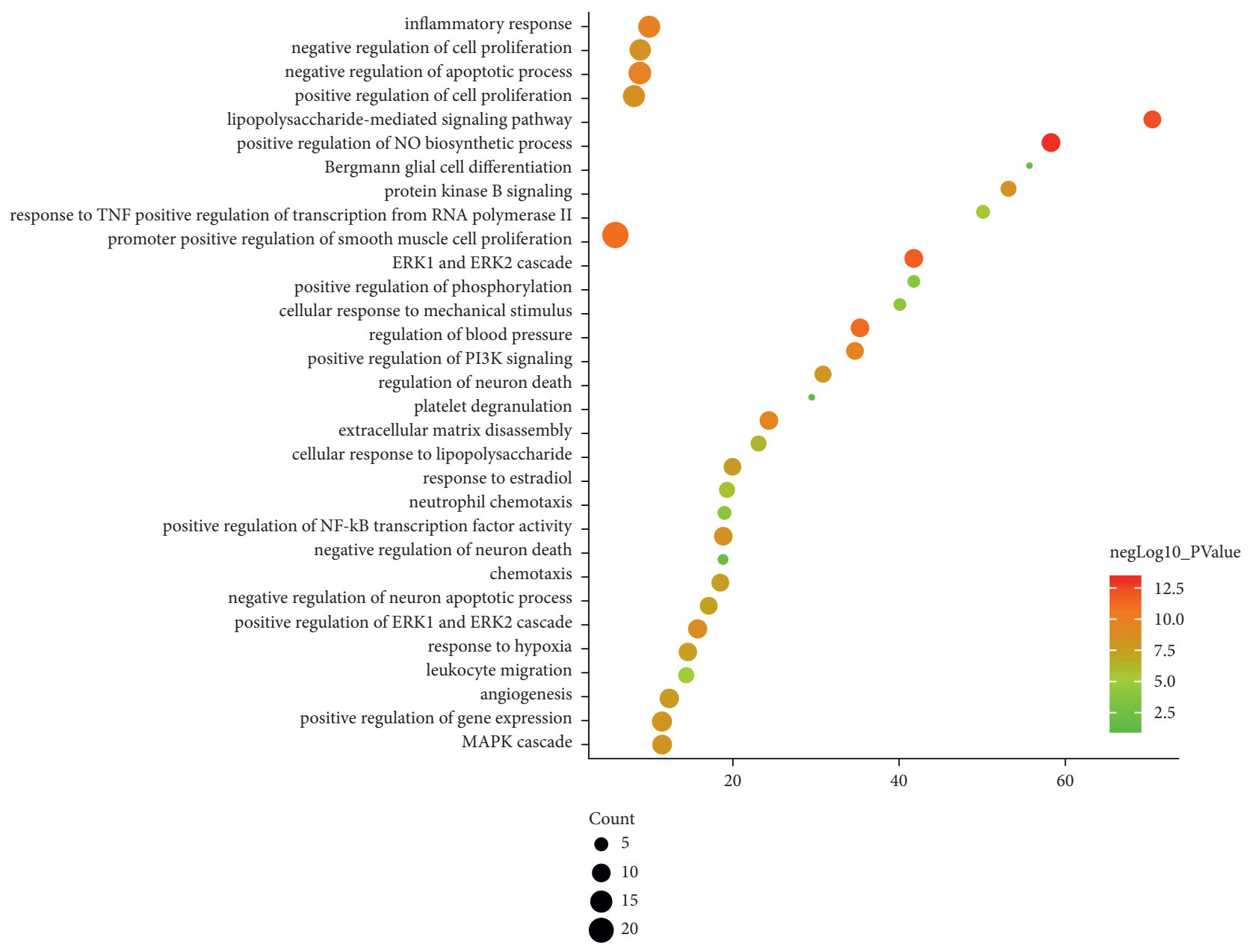

Figure 7: Bubble chart of biological processes (The $X$-axis is fold enrichment.).

The main biological processes, signaling pathways, and reactome pathways are shown in Figures 7,9 , and 10, respectively. Current studies have found that the occurrence and development of CI have slowed blood flow and vascular sclerosis in the cerebral vessels [55]. Astrocytes, endothelial cells, and pericytes constitute the neurovascular units required for neuronal metabolism [56, 57]. When CI occurs, the neurovascular unit is abnormal: hypoxia results in the dysfunction of the endothelial cell barrier of the blood-brain barrier, leading to a decrease in intracellular cAMP levels and an increase in vascular permeability $[58,59]$. Ischemia and reperfusion cause various cells in the neurovascular unit to initiate cell death programs, including apoptosis, autophagy-related cell death, iron death, cell scoring, and necrosis [60, 61]. During CI, these cells contribute to postischemic inflammation at multiple stages of the ischemic cascade [62]. In the inflammatory response, microglia and astrocytes and infiltrating immune cells release a variety of inflammatory factors, including cytokines, chemokines, enzymes, and free radicals, which not only cause brain damage but also affect brain tissue repair $[63,64]$. Recent studies have also shown that anti-inflammatory is an important treatment strategy for CI [65]. After thrombolysis, oxidative stress becomes the central link in cerebral ischemia-reperfusion. During reperfusion, oxygen is replenished, which is essential for maintaining the viability of neurons [66]. However, prooxidase and mitochondria also use oxygen as a substrate, and a large amount of oxygen free radicals (oxidants) are generated during reperfusion. Endogenous antioxidant enzymes, including SOD, can clear oxidants and reduce oxidantinduced brain damage $[67,68]$.

The network pharmacology strategy was used above to predict the mechanism of Radix Rhei Et Rhizome intervention in CI. In order to further explore it, the previous proteomics data were analyzed in depth. The proteomics data come from reference [69].

\subsection{Bioinformatics Analysis of Proteomics Proteins}

3.3.1. Proteomics Proteins' PPI Network. The proteomics proteins of reference [69] are shown in Table S2. A total of 76 proteins were input into String for PPI data. The proteomics proteins' PPI data were composed of 76 proteomics protein nodes and 182 edges (Figure 12).

3.3.2. Enrichment Analysis Results. DAVID and Metascape (http://metascape.org/gp/index.html\#/main/step1) were utilized to analyze the proteins in the proteomics proteins' 


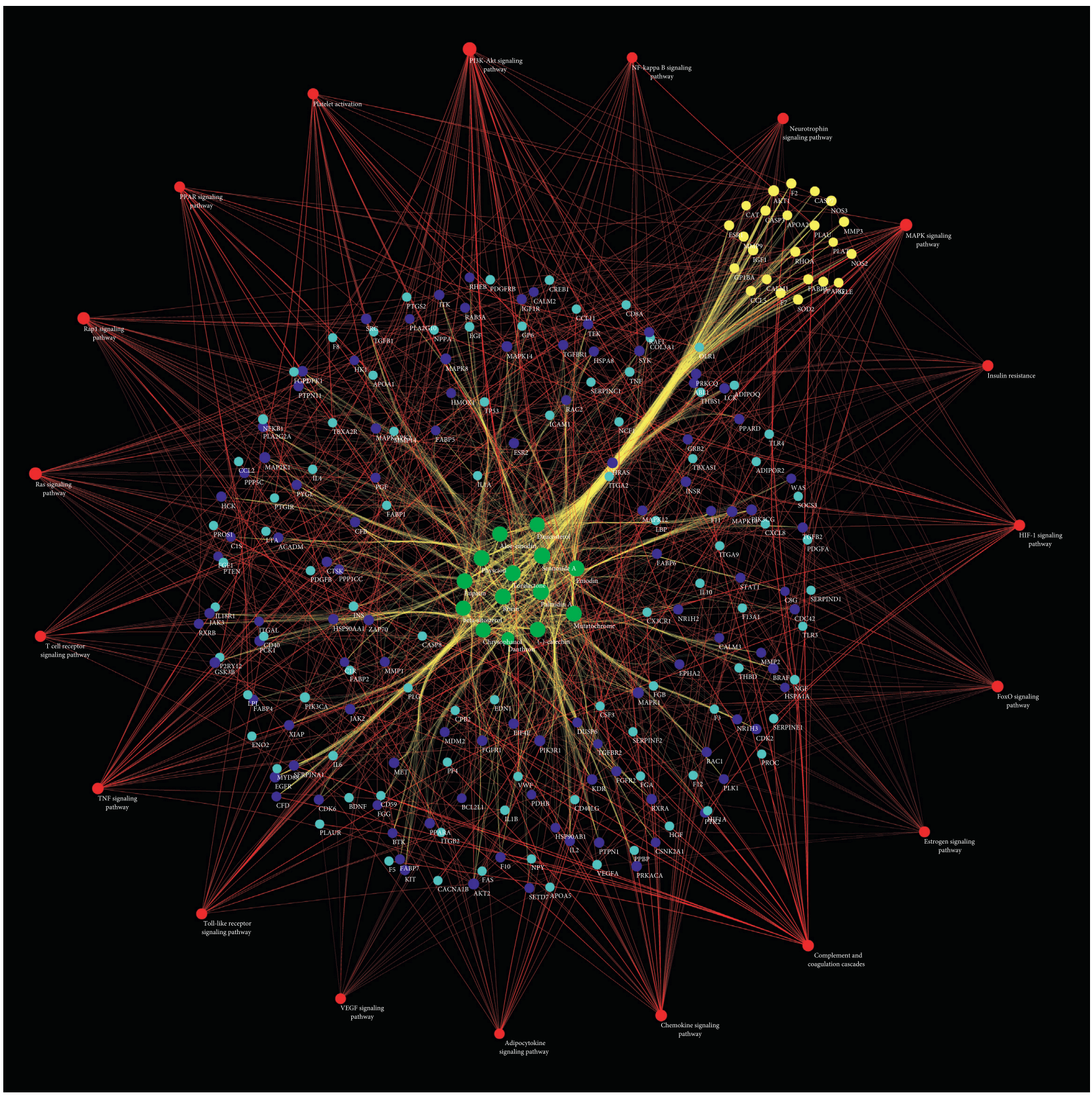

FIGURE 8: Signaling pathways of Radix Rhei Et Rhizome-CI PPI network (Red circles stand for signaling pathways. Dark blue circles stand for Radix Rhei Et Rhizome targets. Light blue circles stand for CI genes. Yellow circles stand for Radix Rhei Et Rhizome-CI targets. Green circles stand for Radix Rhei Et Rhizome compounds. The larger the node size, the higher the degree of the node. The thicker the line, the greater the edge betweenness of the node.).

PPI network (Figure 13). The details of biological processes, signaling pathways, and reactome pathways are shown in Table S7. The clusters are shown in Figure 14.

The top 20 proteins in Figure 12 were Syp, Syn1, Mbp, Gap43, Aif1, Camk2a, Syt1, Calm1, Calb1, Nsf, Nefl, Hspa5, Nefh, Ncam1, Dcx, Unc13a, Mapk1, Syt2, Dnm1, and Cltc. These proteins may be the key targets of Radix Rhei Et Rhizome in the treatment of CI. As a specific marker protein of synaptic vesicles, synaptophysin (SYP) is a sign of synapse occurrence. Its density and distribution can indirectly reflect the number and distribution of synapses in the body [70, 71]. Studies have found that after CI in rats, the synaptophysin immune response was significantly enhanced compared with the sham operation group, and the expression reached a peak at 2 weeks after cerebral ischemia, and it significantly decreased at 3 weeks [72]. This experimental study found that after Radix Rhei Et Rhizome treatment, its expression was significantly increased compared with the model group, indicating that Radix Rhei Et Rhizome may resist synapse damage or promote synaptic regeneration after cerebral ischemia. Synapse protein I gene (SYN1) mainly mediates the delivery of synaptic vesicles and circulation [73] and plays an important role in neurodegenerative diseases [74], such as Alzheimer's disease, Parkinson's disease, and 


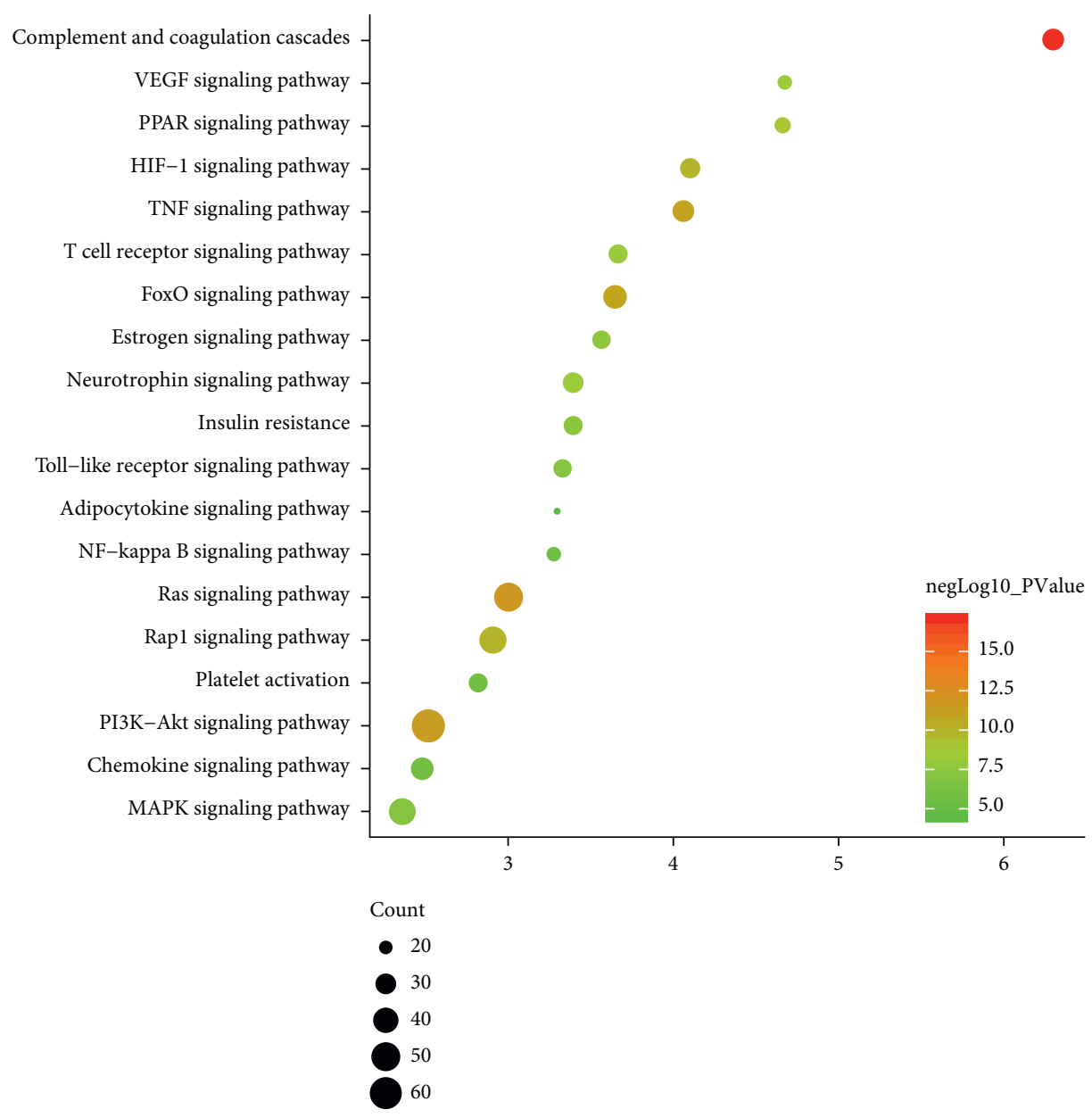

FIgURE 9: Signaling pathways of biological processes (The $X$-axis is fold enrichment.).

TABLE 2: The number of targets regulated by the components of Radix Rhei Et Rhizome.

\begin{tabular}{lc}
\hline Components & Number of targets \\
\hline Sennoside A & 103 \\
Palmidin A & 99 \\
Emodin & 98 \\
Toralactone & 96 \\
Mutatochrome & 96 \\
Rhein & 95 \\
Physcion & 95 \\
Eupatin & 93 \\
(-)-Catechin & 91 \\
Aloe-emodin & 88 \\
Chrysophanol & 86 \\
Beta-sitosterol & 85 \\
Daucosterol & 85 \\
Danthron & 53 \\
\hline
\end{tabular}

amyotrophic lateral sclerosis. This study suggests that SYN1 may be a potential drug target for neurodegenerative diseases caused by CI, among which Radix Rhei Et Rhizome can reduce CI by interfering with SYN1.

GAP-43 is a calmodulin-binding phosphoprotein that has been isolated and identified in recent years [75]. In the process of neuron development and regeneration, GAP- 43 is synthesized in large quantities along with the growth of axons and is a marker of axon growth. The expression product is mainly located on the plasma membrane surface of the axon growth cone [76]. Studies have shown that the increase in GAP- 43 content in the penumbra of the ischemic penumbra of one middle cerebral artery 3 to 14 days after the embolization of the middle cerebral artery is synchronized with the recovery of the function of the affected limb [77]. Another study found that the expression of GAP-43 in the brain increased significantly after cerebral infarction, peaked at 1 week after ischemia-reperfusion, and began to decrease after 2 weeks $[78,79]$. This experiment found that Radix Rhei Et Rhizome can significantly enhance the expression of GAP43 in the cortical ischemic penumbra, suggesting that Radix Rhei Et Rhizome can effectively promote the regeneration of axons in CI model rats and induce the recovery of nerve function.

MBP is mainly located on the serosal surface of the myelin sheath and is the main protein of the myelin sheath of the central nervous system. Its main function is to maintain the integrity and functional stability of the myelin sheath of the central nervous system, and its neural tissue specificity is high [80]. When acute cerebral infarction (ACI) occurs, when the human central nervous system is damaged, the blood-brain barrier function is unbalanced, which increases 


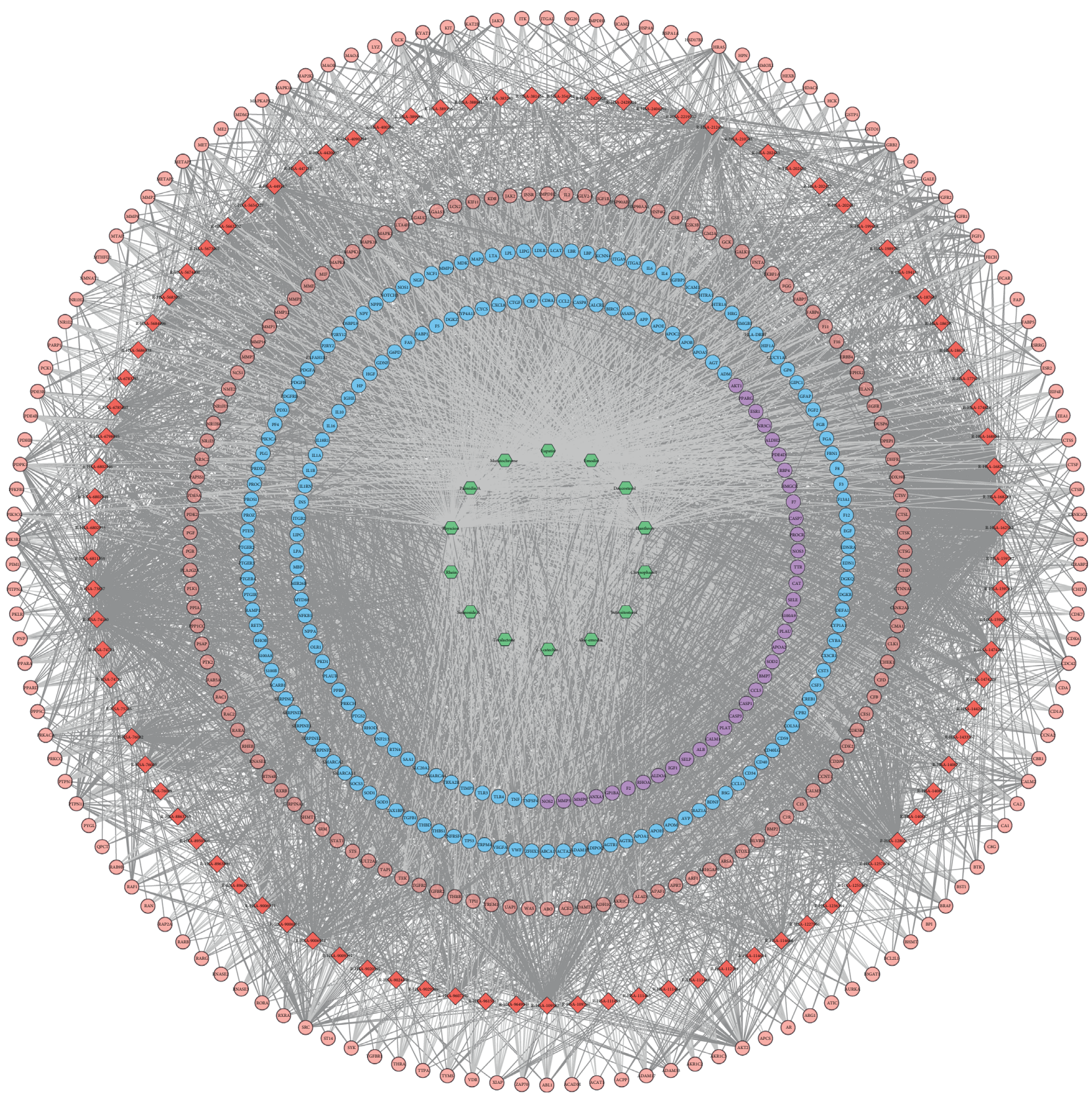

FIgURE 10: Reactome pathways of Radix Rhei Et Rhizome-CI PPI Network (Green hexagons stand for potential compounds. Blue, pink, and purple circles stand for CI genes, Radix Rhei Et Rhizome targets, and Radix Rhei Et Rhizome-CI targets, respectively. Red diamonds stand for reactome pathways. Dark lines stand for relationships among reactome pathways and targets. Light lines stand for relationships among herbs and targets.).

its permeability, and MBP can easily pass through the bloodbrain barrier and be released into the blood. At present, the detection of serum NSE and MBP expression levels is of great value in assessing the prognosis of ACI disease [81]. The results of this study showed that the MBP level of the CI group increased rapidly and the MBP level decreased after Radix Rhei Et Rhizome treatment, suggesting that Radix Rhei Et Rhizome treatment of the ACI rat model can promote the disease outcome and reduce the MBP level.

Allogeneic inflammatory factor-1 (AIF-1) is a $17 \mathrm{kDa}$ cytoplasmic calcium-binding inflammatory response scaffold protein, which is mainly expressed in immune cells [82].
AIF-1 affects the immune system at several key points, thereby regulating inflammatory diseases [83]. AIF-1 promotes the expression of inflammatory mediators such as cytokines, chemokines, and inducible nitric oxide synthase (iNOS) and promotes the proliferation and migration of inflammatory cells. Current research shows that it regulates central nervous system (CNS) damage [82, 83]. The results of this study showed that the level of cerebral infarction (AIF1) increased rapidly, and the level of AIF-1 decreased after Radix Rhei Et Rhizome treatment. It is suggested that Radix Rhei Et Rhizome can promote the outcome of CI and reduce the level of inflammation. 


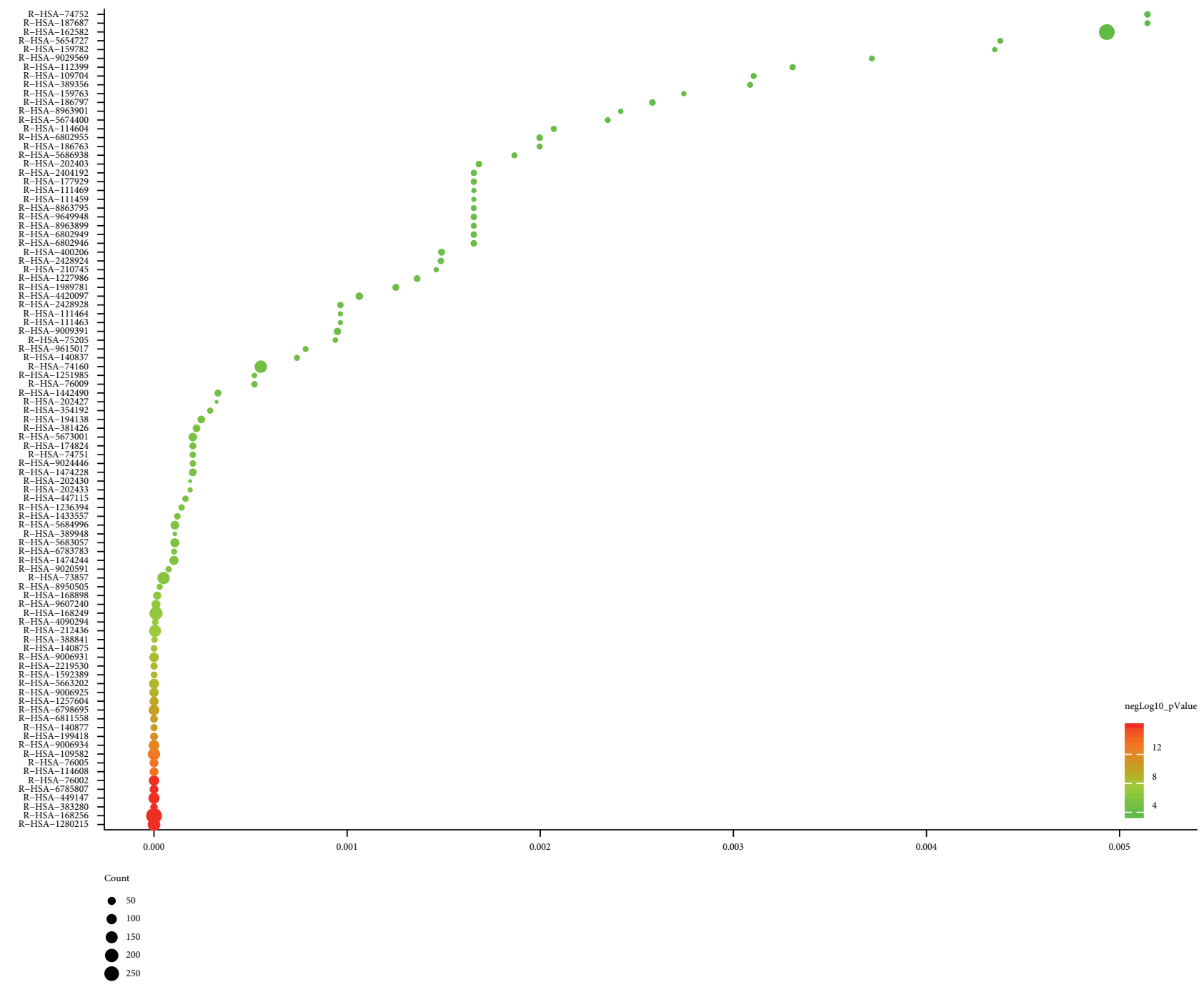

FIgURE 11: Reactome pathways of biological processes (The $X$-axis is FDR.).

TABLE 3: The number of targets regulated by the components of Radix Rhei Et Rhizome.

\begin{tabular}{lc}
\hline Components & Number of targets \\
\hline Palmidin A & 222 \\
Sennoside A & 213 \\
Toralactone & 212 \\
Emodin & 209 \\
Rhein & 209 \\
Eupatin & 207 \\
(-)-Catechin & 205 \\
Aloe-emodin & 202 \\
Physcion & 202 \\
Mutatochrome & 193 \\
Chrysophanol & 178 \\
Beta-sitosterol & 171 \\
Daucosterol & 168 \\
Danthron & 116 \\
\hline
\end{tabular}

The alpha (CAMK2A) of calcium/calmodulin-dependent protein kinase II (CaMKII) plays a key role in neuronal plasticity and brain learning and memory [84, 85]. After Radix Rhei Et Rhizome intervention, the expression of
CAMK2A was upregulated. The main biological function of Syt1 is to trigger vesicle fusion [86], which is related to the molecular mechanism of neuronal endocytosis and exocytosis coupling [87]. This study found that Syt1 was upregulated after Radix Rhei Et Rhizome intervention. Calmodulin 1 (CALM1) is highly expressed in the human brain tissue, and its biological function is mainly related to axon transmission [88]. In addition, the Calm1 signaling pathway is crucial for the migration of precerebellar neurons in mice [89]. Calm1-L plays a functional role in the central and peripheral nervous system [90]. This study found that Calm1 was upregulated after Radix Rhei Et Rhizome intervention. A recent study also showed that CALM1 rs3179089 gene polymorphism is associated with CI in Chinese Han population [91].

Calbindin 1 (Calb1) acts as a buffer, sensor, and transporter of intracellular $\mathrm{Ca}^{2+}$. Different types of hippocampal neurons have different Calb1 concentrations. Since Calb1 can inhibit the increase of free $\mathrm{Ca}^{2+}$, it accelerates the collapse of the $\mathrm{Ca}^{2+}$ gradient after the influx of $\mathrm{Ca}^{2+}$ stops [92, 93]. Current research shows that it plays a role in neurotransmitter and hormone release, neuron differentiation, brain wiring, and neuron development [94, 95]. The 


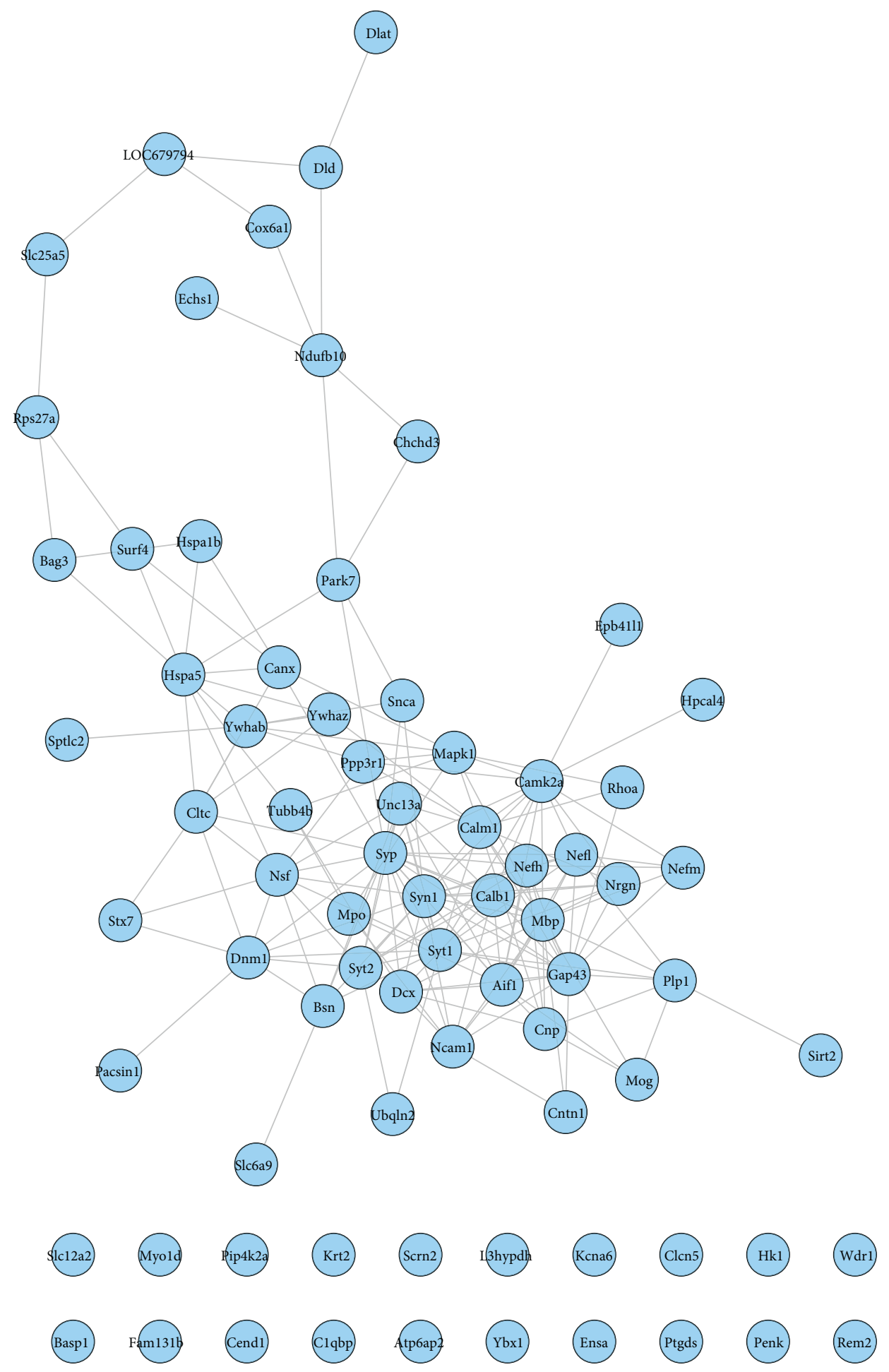

FIGURE 12: Proteomics proteins' PPI network.

results of this study showed that the level of Calb1 was downregulated in the CI group, and the level of Calb1 was upregulated after Radix Rhei Et Rhizome treatment. It is suggested that Radix Rhei Et Rhizome may promote disease outcome through neurotransmitter and other methods.

$\mathrm{N}$-ethylmaleimide-sensitive fusion protein (NSF) is an ATPase that plays an important role in intracellular membrane vesicle transport [96]. It is highly conservative in evolution and participates in the secretion process of different species and different cell types [96, 97]. Current research shows that it plays an important role in the process of neurotransmitter release by synaptic vesicle exocytosis at presynaptic nerve terminals $[97,98]$. The results of this study showed that the level of NSF in the cerebral infarction group was downregulated and the level of NSF was upregulated after treatment. 


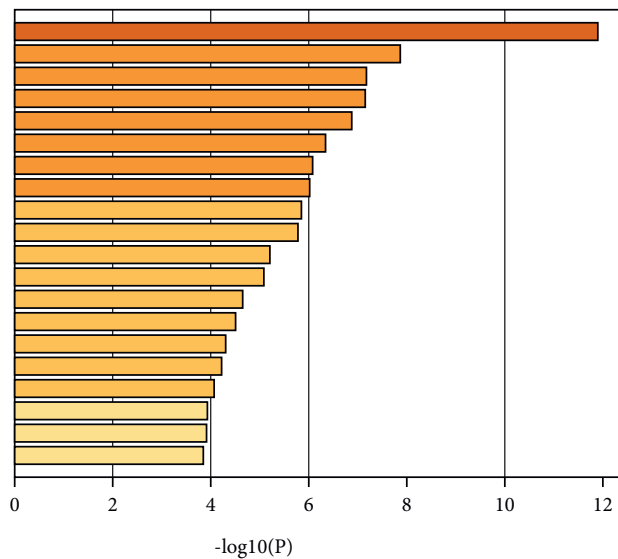

GO:0099504: synaptic vesicle cycle

GO:0061024: membrane organization

GO:0031623: receptor internalization

GO:0033693: neurofilament bundle assembly

R-RNO-373760: L1CAM interactions

R-RNO-3858494: Beta-catenin independent WNT signaling

R-RNO-8856828: Clathrin-mediated endocytosis

R-RNO-112315: Transmission across Chemical Synapses

GO:0072347: response to anesthetic

GO:0007568: aging

GO:0006119: oxidative phosphorylation

R-RNO-450531: Regulation of mRNA stability by proteins that bind AU-rich elements

GO:0010720: positive regulation of cell development

GO:0097201: negative regulation of transcription from RNA polymerase II promoter in response to stress

GO:0021700: developmental maturation

GO:0043269: regulation of ion transport

GO:0007626: locomotory behavior

GO:0045744: negative regulation of $\mathrm{G}$ protein-coupled receptor signaling pathway

rno05010: Alzheimer's disease

R-RNO-2262752: Cellular responses to stress

(a)
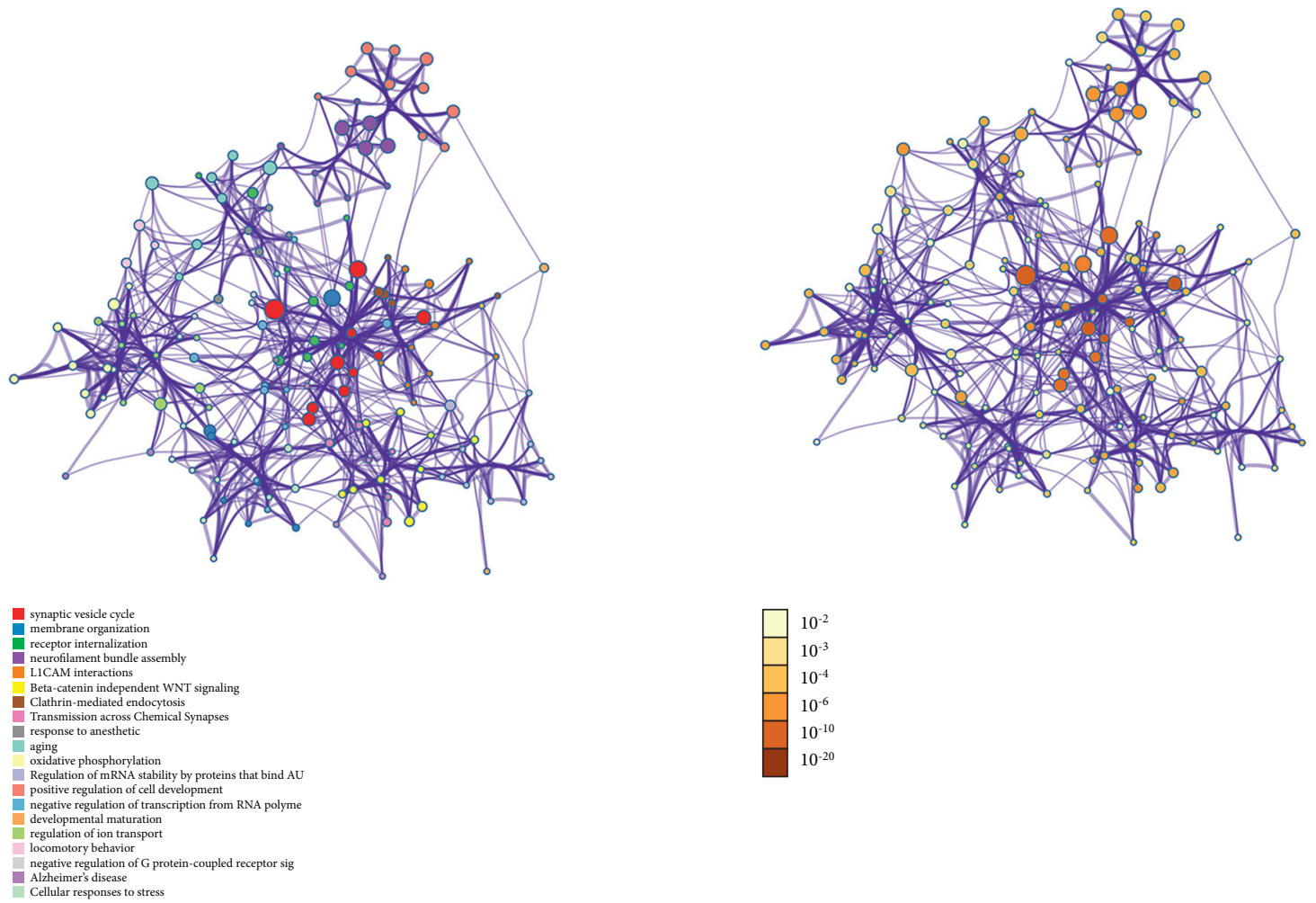

(b)

FIgURe 13: The Metascape results: (a) top biological processes, signaling pathways, and reactome pathways of the proteomics proteins' PPI network and (b): PPI networks colored by enrichment results or $P$-values.

Neurofilament light chain (Nefl) belongs to one of the main subtypes of neurofilament protein. It is the main cytoskeleton structural protein of neurons, which is distributed in axons $[99,100]$. Nefl is also an important indicator for judging acute axonal injury [99]. In clinical studies of relapsing multiple sclerosis, Nefl is used as an effective evaluation index for drug anti-inflammatory therapy [101]. Recent studies have shown that emodin activates mTOR and Notch pathways in hypoxic PC12 cells by inhibiting Nefl [102]. In this study, the expression level of Nefl in CI model rats was higher but decreased after intervention, indicating that there is acute axon damage in acute stroke and Radix Rhei Et Rhizome may be able to protect axons.
The endoplasmic reticulum stress chaperone protein HSPA5 is mainly related to endoplasmic reticulum stress. Current studies have shown that endoplasmic reticulum stress can induce autophagy activation [103]. Previous studies have confirmed that in the mouse brain I/R model, the expression of HSPA5 protein is increased, and it has a neuroprotective effect [104]. At present, by injecting HSPA5 siRNA into the anterior ventricle of CI mice, the expression of LC3-1/LC3-I is significantly reduced, and it will also lead to the loss of nerve cells in the cerebral ischemic cortex of mice and aggravation of neurobehavioral damage. This is similar to the effect of the autophagy inhibitor 3-MA, indicating that HSPA5-mediated autophagy may play a 

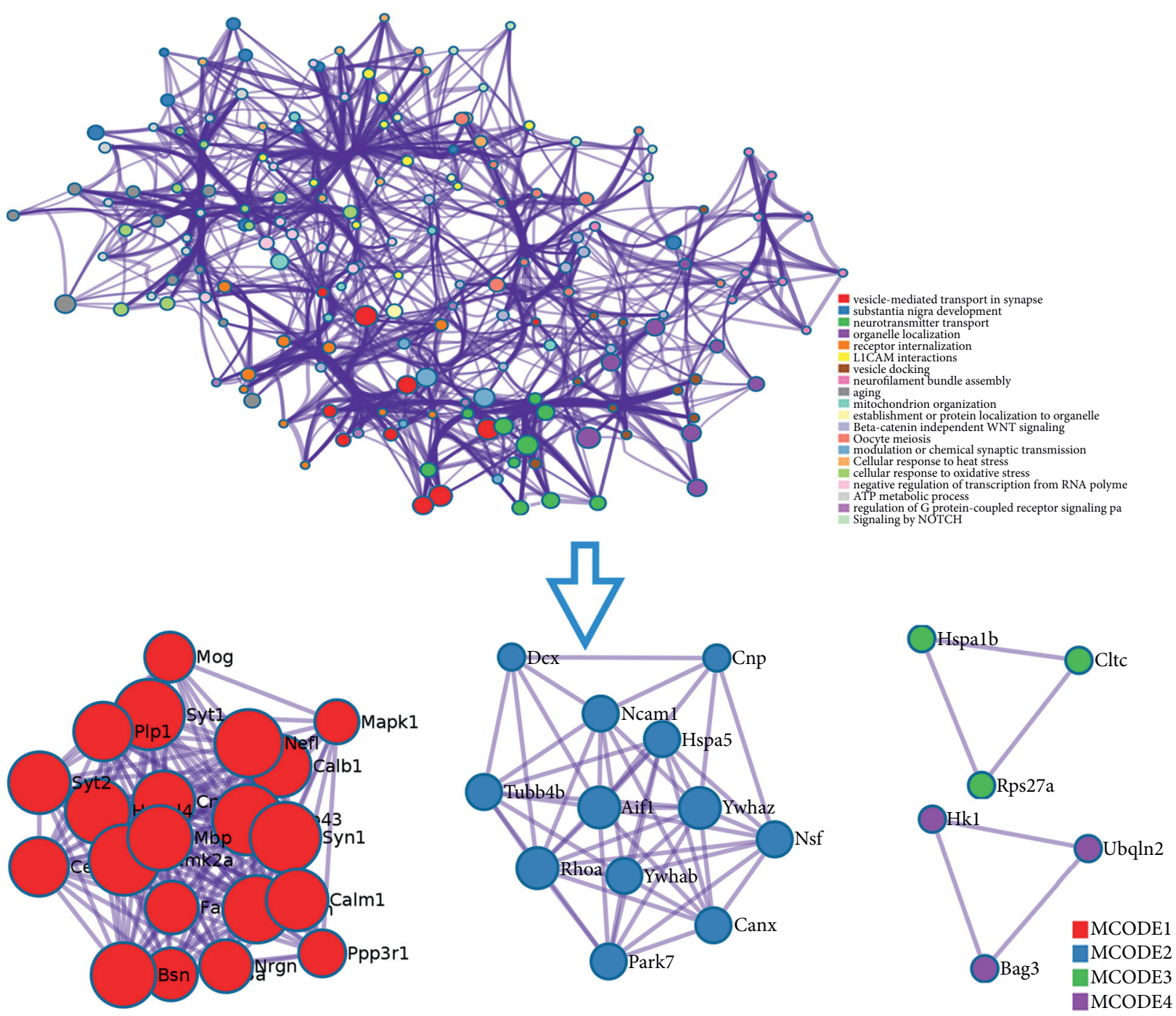

FIGURE 14: Clusters of proteomics proteins' PPI network.

neuroprotective effect in mouse I/R $[105,106]$. The results of this study showed that the level of HSPA5 in the CI group was downregulated, but after treatment, the level of HSPA5 was upregulated.

NCAM1 is a member of the cell adhesion molecule family and is a molecular cleavage of the immunoglobulin superfamily. NCAM1 is a membrane protein that includes three subtypes: NCAM-120, NCAM-140, and NCAM-180. It is mainly expressed in the nervous system and is involved in regulating the function of nerve cells and neuron migration [107]. NCAM1 is expressed in neural stem cells. In addition, astrocytes also express many adhesion molecules, such as VCAM1, NCAM1, and ICAM1, which represent many potential drug targets for inflammatory diseases of the central nervous system [108-110]. This study showed that the level of NCAM1 in the CI group was downregulated. After Radix Rhei Et Rhizome treatment, the level of NCAM1 was upregulated, suggesting that Radix Rhei Et Rhizome may inhibit adhesion molecules and is related to inflammatory factors.

Dcx is a microtubule-associated phosphoprotein, which is specifically expressed in newborn neuroblasts and immature neurons in DG $[111,112]$. Therefore, Dcx has been widely used to label the cell bodies, processes, and growth cones of newborn neurons. Studies have reported that after CI, Dcx strengthens the differentiation of nerve cells in the DG area of the hippocampus and promotes the rehabilitation of nerve function [113]. This study showed that the level of Dcx in the CI group was downregulated, and after treatment, the level of Dcx was upregulated, suggesting that Radix Rhei Et Rhizome may regulate the generation of new neurons and promote the outcome of CI.

The limitation of this study is that although the pharmacokinetic parameters are used to predict the composition of Radix Rhei Et Rhizome and the composition was supplemented as much as possible by searching the literature, due to the limitations of the current detection technology, there are still active ingredients that may not be included. Since the intestinal flora may metabolize and secondary modify the active components of Radix Rhei Et Rhizome, these components may be traced in the blood. In the future, better technology is needed to detect these components. In 
addition, although this study analyzed the main active components of Radix Rhei Et Rhizome in the treatment of CI through chemoinformatics and explored its possible synergistic effects, there is still a lack of in vivo and in vitro experiments related to their intervention in CI. In the future, we will explore the synergistic compatibility of these components in the $\mathrm{CI}$ in vitro model and the $\mathrm{CI}$ in vivo model and look forward to further development of new drugs for the treatment of CI, laying the foundation for its clinical application.

Our previous research evaluated the therapeutic effect of Radix Rhei Et Rhizome on cerebral hemorrhage [114], while this study explored the mechanism of Radix Rhei Et Rhizome in the treatment of CI, and this study found that Radix Rhei Et Rhizome may regulate the synaptic remodeling and the regeneration of nerve cell axons after cerebral ischemia. Compared with previous research [114], this study explored the mechanism of Radix Rhei Et Rhizome intervention in CI. This study found that Radix Rhei Et Rhizome may treat CI through biological process (such as platelet degranulation, cell migration, fibrinolysis, platelet activation, hypoxia, angiogenesis, endothelial cell apoptosis, coagulation, and neuronal apoptosis), signaling pathways (such as Ras, PI3K-Akt, TNF, FoxO, HIF-1, and Rap1), and reactome pathways (such as inflammatory cytokines, platelet activation, response to elevated platelet cytoplasmic $\mathrm{Ca}^{2+}$, and hemostasis).

\section{Conclusion}

Radix Rhei Et Rhizome may play the therapeutic role for CI through regulating biological modules such as synaptic vesicles and neurotransmitter secretion and transport, energy metabolism, neuronal programmed death (apoptotic autophagy) module, calcium ion regulation of exocytosis and cytoplasmic calcium ion release, endoplasmic reticulum oxidative stress, and neuroplasticity (neuron and synaptic plasticity).

\section{Data Availability}

The data used to support the findings of this study are included within the article and the supplementary information files.

\section{Conflicts of Interest}

The authors declare no competing interests.

\section{Authors' Contributions}

Jinsong Zeng, Xiaofei Zhu, and Liang Liu are responsible for the study concept and design. Jinsong Zeng, Xiaofei Zhu, Mengxia Yuan, Jiamin $\mathrm{Wu}$, Wang Xiang, Yonghe $\mathrm{Wu}$, Zhiyong Long, and Liang Liu are responsible for data analysis and interpretation. Jinsong Zeng and Xiaofei Zhu drafted the paper. Liang Liu supervised the study. All the authors participated in the analysis and interpretation of data and approved the final paper. Wang Xiang and Zhiyong Long should be considered joint first authors.

\section{Acknowledgments}

This research was supported by the Natural Science Foundation of Hunan Province (2020JJ5442) and Open Fund for First-class Discipline of Integrated Traditional Chinese and Western Medicine of the Hunan University of Chinese Medicine (2020ZXYJH09).

\section{Supplementary Materials}

Table S1: potential targets for potential compounds; Table S2: proteomics data; Table S3: CI gene; Table S4: enrichment analysis of clusters based on gene ontology (GO) annotation of Radix Rhei Et Rhizome-CI PPI network; Table S5: pathway enrichment analysis of Radix Rhei Et Rhizome-CI PPI network; Table S6: reactome pathways of Radix Rhei Et Rhizome-CI PPI network; and Table S7: the biological processes, signaling pathways, and reactome of proteomics proteins' PPI network. (Supplementary Materials)

\section{References}

[1] H. Slyter, "Guidelines for the management of patients with acute ischemic stroke," Stroke, vol. 26, no. 1, pp. 137-138, 1995.

[2] Z. Wang, S. Hu, S. Sang, L. Luo, and C. Yu, "Age-periodcohort analysis of stroke mortality in China," Stroke, vol. 48, no. 2, pp. 271-275, 2017.

[3] J. Cao, E. S. Eshak, K. Liu, K. Gero, Z. Liu, and C. Yu, “Ageperiod-cohort analysis of stroke mortality attributable to high sodium intake in China and Japan," Stroke, vol. 50, no. 7, pp. 1648-1654, 2019.

[4] R. A. Radu, E. O. Terecoasă, O. A. Băjenaru, and C. Tiu, "Etiologic classification of ischemic stroke: where do we stand?" Clinical Neurology and Neurosurgery, vol. 159, pp. 93-106, 2017.

[5] H. K. Eltzschig and T. Eckle, "Ischemia and reperfusion-from mechanism to translation," Nature Medicine, vol. 17, no. 11, pp. 1391-1401, 2011.

[6] T. Kalogeris, C. P. Baines, M. Krenz, and R. J. Korthuis, "Cell biology of ischemia/reperfusion injury," International Review of Cell and Molecular Biology, vol. 198, pp. 229-317, 2012.

[7] W. Cai, H. Liu, J. Zhao et al., "Pericytes in brain injury and repair after ischemic stroke," Translational Stroke Research, vol. 8, no. 2, pp. 107-121, 2017.

[8] J. H. Choi and J. Pile-Spellman, "Reperfusion changes after stroke and practical approaches for neuroprotection," Neuroimaging Clinics of North America, vol. 28, no. 4, pp. 663-682, 2018.

[9] J. Yang, M. Chen, R. Y. Cao, Q. Li, and F. Zhu, "The role of circular RNAs in cerebral ischemic diseases: ischemic stroke and cerebral ischemia/reperfusion injury," Advances in Experimental Medicine and Biology, vol. 1087, no. 2, pp. 309325, 2018.

[10] C. Hentia, A. Rizzato, E. Camporesi et al., "An overview of protective strategies against ischemia/reperfusion injury: the role of hyperbaric oxygen preconditioning," Brain and Behavior, vol. 8, no. 5, Article ID e00959, 2018.

[11] B. P. Gaire, "Herbal medicine in ischemic stroke: challenges and prospective," Chinese Journal of Integrative Medicine, vol. 24, no. 4, pp. 243-246, 2018. 
[12] P.-f. Wu, Z. Zhang, F. Wang, and J.-g. Chen, "Natural compounds from traditional medicinal herbs in the treatment of cerebral ischemia/reperfusion injury," Acta Pharmacologica Sinica, vol. 31, no. 12, pp. 1523-1531, 2010.

[13] N. Ghosh, R. Ghosh, Z. A. Bhat et al., "Advances in herbal medicine for treatment of ischemic brain injury," Natural product communications, vol. 9, no. 7, pp. 1045-1055, 2014.

[14] A.-j. Liu, L. Song, Y. Li et al., "Active compounds of rhubarb root and Rhizome in animal model experiments of focal cerebral ischemia," Evidence-Based Complementary and Alternative Medicine, vol. 2015, Article ID 210546, 13 pages, 2015.

[15] Q. Guan, S. Liang, Z. Wang, Y. Yang, and S. Wang, "1H NMR-based metabonomic analysis of the effect of optimized rhubarb aglycone on the plasma and urine metabolic fingerprints of focal cerebral ischemia-reperfusion rats," Journal of Ethnopharmacology, vol. 154, no. 1, pp. 65-75, 2014.

[16] R.-R. Li, X.-F. Liu, S.-X. Feng et al., "Pharmacodynamics of five anthraquinones (aloe-emodin, emodin, rhein, chysophanol, and physcion) and reciprocal pharmacokinetic interaction in rats with cerebral ischemia," Molecules, vol. 24, no. 10, p. 1898, 2019.

[17] N. Zhang, X. Zhang, X. Liu et al., "Chrysophanol inhibits NALP3 inflammasome activation and ameliorates cerebral ischemia/reperfusion in mice," Mediators of Inflammation, vol. 2014, Article ID 370530, 12 pages, 2014.

[18] W.-T. Yang, Y. Wang, Y.-H. Shi et al., "Herbal compatibility of ginseng and rhubarb exerts synergistic neuroprotection in cerebral ischemia/reperfusion injury of rats," Frontiers in Physiology, vol. 10, p. 1174, 2019.

[19] X. Li, S. Chu, Y. Liu, and N. Chen, "Neuroprotective effects of anthraquinones from rhubarb in central nervous system diseases," Evidence-Based Complementary and Alternative Medicine, vol. 2019, Article ID 3790728, 12 pages, 2019.

[20] T. Bao, K. Yang, Z. Long, L. Zeng, and Y. Li, "Systematic pharmacological methodology to explore the pharmacological mechanism of siwu decoction for osteoporosis," Medical Science Monitor, vol. 25, pp. 8152-8171, 2019.

[21] K. Yang, L. Zeng, T. Bao, Z. Long, and B. Jin, "Exploring the pharmacological mechanism of quercetin-resveratrol combination for polycystic ovary syndrome: a systematic pharmacological strategy-based research," Scientific Reports, vol. 9, no. 1, p. 18420, 2019.

[22] L. Zeng, K. Yang, and J. Ge, "Uncovering the pharmacological mechanism of Astragalus salvia compound on pregnancy-induced hypertension syndrome by a network pharmacology approach," Scientific Reports, vol. 7, no. 1, p. 16849, 2017.

[23] L. Zeng and K. Yang, "Exploring the pharmacological mechanism of Yanghe decoction on HER2-positive breast cancer by a network pharmacology approach," Journal of Ethnopharmacology, vol. 199, pp. 68-85, 2017.

[24] K. Yang, L. Zeng, A. Ge et al., "Investigating the regulation mechanism of baicalin on triple negative breast cancer's biological network by a systematic biological strategy," Biomedicine \& Pharmacotherapy, vol. 118, p. 109253, 2019.

[25] J. Ru, P. Li, J. Wang et al., "TCMSP: a database of systems pharmacology for drug discovery from herbal medicines," Journal of Cheminformatics, vol. 6, no. 1, p. 13, 2014.

[26] F.-P. Chen, C.-M. Chang, S.-J. Hwang, Y.-C. Chen, and F.-J. Chen, "Chinese herbal prescriptions for osteoarthritis in Taiwan: analysis of national health insurance dataset," $B M C$
Complementary and Alternative Medicine, vol. 14, no. 1, p. 91, 2014.

[27] X. Xu, W. Zhang, C. Huang et al., "A novel chemometric method for the prediction of human oral bioavailability," International Journal of Molecular Sciences, vol. 13, no. 6, pp. 6964-6982, 2012.

[28] R. Ano, Y. Kimura, M. Shima, R. Matsuno, T. Ueno, and M. Akamatsu, "Relationships between structure and highthroughput screening permeability of peptide derivatives and related compounds with artificial membranes: application to prediction of Caco-2 cell permeability," Bioorganic \& Medicinal Chemistry, vol. 12, no. 1, pp. 257-264, 2004.

[29] G. X. Hu, C. H. Zhang, W. N. Zhao, and Q. S. Yu, "QSPR study on the permeability of drugs across Caco-2 monolayer," Journal of Zhejiang University, vol. 3, pp. 304-308, 2009.

[30] W. P. Walters and M. A. Murcko, "Prediction of "druglikeness"” Advanced Drug Delivery Reviews, vol. 54, no. 3, pp. 255-271, 2002.

[31] D. Metodiewa, A. Kochman, and S. Karolczak, "Evidence for antiradical and antioxidant properties of four biologically active N,N-diethylaminoethyl ethers of flavaone oximes: a comparison with natural polyphenolic flavonoid rutin action," IUBMB Life, vol. 41, no. 5, pp. 1067-1075, 1997.

[32] T. Pang, Z. Xie, Y. Chen, Y. H. Li, and M. R. Wen, "Simultaneous determination of 7 anthraquinone compounds in rhubarb compatible drugs by HPLC," Chinese Journal of New Drugs, vol. 26, no. 9, pp. 1079-1088, 2017, in Chinese.

[33] X. Y. Gao and J. Q. Lu, "Simultaneous determination of seven anthraquinones in rhubarb by HPLC-DAD," Journal of Pharmaceutical Analysis, vol. 30, no. 4, pp. 1636-1641, 2010, in Chinese.

[34] X. Wang, Y. Shen, S. Wang et al., "PharmMapper 2017 update: a web server for potential drug target identification with a comprehensive target pharmacophore database," Nucleic Acids Research, vol. 45, no. W1, pp. W356-W360, 2017.

[35] G. Stelzer, R. Rosen, I. Plaschkes et al., "The GeneCards suite: from gene data mining to disease genome sequence analysis," Current Protocols in Bioinformatics, vol. 54, pp. 1.30.11.30.33, 2016.

[36] A. Hamosh, A. F. Scott, J. S. Amberger, C. A. Bocchini, and V. A. McKusick, "Online Mendelian Inheritance in Man (OMIM), a knowledge base of human genes and genetic disorders," Nucleic Acids Research, vol. 33, no. Database issue, pp. D514-D517, 2004.

[37] D. Szklarczyk, A. Franceschini, S. Wyder et al., "STRING v10: protein-protein interaction networks, integrated over the tree of life," Nucleic Acids Research, vol. 43, no. D1, pp. D447-D452, 2015.

[38] G. D. Bader and C. W. Hogue, "An automated method for finding molecular complexes in large protein interaction networks," BMC Bioinformatics, vol. 4, no. 1, pp. 2-57, 2003.

[39] D. W. Huang, B. T. Sherman, and R. A. Lempicki, "Systematic and integrative analysis of large gene lists using DAVID bioinformatics resources," Nature Protocols, vol. 4, no. 1, pp. 44-57, 2009.

[40] A. Fabregat, S. Jupe, L. Matthews et al., "The reactome pathway knowledgebase," Nucleic Acids Research, vol. 46, no. D1, pp. D649-D655, 2018.

[41] Y. Li, Q.-Q. Xu, C.-S. Shan, Y.-H. Shi, Y. Wang, and G.-Q. Zheng, "Combined use of emodin and ginsenoside $\mathrm{Rb} 1$ exerts synergistic neuroprotection in cerebral ischemia/ 
reperfusion rats," Frontiers in Pharmacology, vol. 9, p. 943, 2018.

[42] J.-S. Lu, J.-X. Liu, W.-Y. Zhang, S.-W. Liang, D. Wang, and J. Fang, "Preventive effects of Emodin on cerebral ischemia injury and expression of the inflammatory factors in rats with cerebral ischemia," China Journal of Chinese Materia Medica, vol. 30, no. 24, pp. 1939-1943, 2005.

[43] S. M. Ahn, H. N. Kim, Y. R. Kim et al., "Emodin from Polygonum multiflorum ameliorates oxidative toxicity in HT22 cells and deficits in photothrombotic ischemia," Journal of Ethnopharmacology, vol. 188, pp. 13-20, 2016.

[44] T. Liu, H.-T. Hu, and Q.-R. Sun, "Neuroprotective effects of emodin on primary rat cortical neurons apoptosis induced by hydrogen peroxide," Zhong Yao Cai, vol. 33, no. 7, pp. 1116-1119, 2010.

[45] H. Guo, X. Shen, Y. Xu, J. Yuan, D. Zhao, and W. Hu, "Emodin prevents hypoxic-ischemic neuronal injury: involvement of the activin a pathway," Neural Regeneration Research, vol. 8, no. 15, pp. 1360-1367, 2013.

[46] A. B. Belousov, J. D. Fontes, M. Freitas-Andrade, and C. C. Naus, "Gap junctions and hemichannels: communicating cell death in neurodevelopment and disease," BMC Cell Biology, vol. 18, no. S1, p. 1, 2017.

[47] Y. Zhao, Y. Fang, J. Li et al., "Neuroprotective effects of chrysophanol against inflammation in middle cerebral artery occlusion mice," Neuroscience Letters, vol. 630, pp. 16-22, 2016.

[48] H. Ageta, A. Murayama, R. Migishima et al., "Activin in the brain modulates anxiety-related behavior and adult neurogenesis," PLoS One, vol. 3, no. 4, p. e1869, 2008.

[49] S. W. Leung, J. H. Lai, J. C.-C. Wu et al., "Neuroprotective effects of emodin against ischemia/reperfusion injury through activating ERK-1/2 signaling pathway," International Journal of Molecular Sciences, vol. 21, no. 8, pp. 2899-2020, 2020.

[50] Q. Zhao, X. Wang, A. Chen et al., "Rhein protects against cerebral ischemic/reperfusion induced oxidative stress and apoptosis in rats," International Journal of Molecular Medicine, vol. 41, no. 5, pp. 2802-2812, 2018.

[51] Y. Zhao, Y. Huang, Y. Fang et al., "Chrysophanol attenuates nitrosative/oxidative stress injury in a mouse model of focal cerebral ischemia/reperfusion," Journal of Pharmacological Sciences, vol. 138, no. 1, pp. 16-22, 2018.

[52] Y. Zhao, Y. Fang, H. Zhao et al., "Chrysophanol inhibits endoplasmic reticulum stress in cerebral ischemia and reperfusion mice," European Journal of Pharmacology, vol. 818, no. 5, pp. 1-9, 2018.

[53] J. Li, J. Liu, D. Wang et al., "Effects of rhubarb aglycone combined with thrombolysis on brain microvascular basement membrane impairment in rats with thrombus-occluded cerebral ischemia," Zhongguo Zhongyao Zazhi, vol. 35, no. 21, pp. 2908-2911, 2010.

[54] S.-X. Feng, J.-S. Li, L.-B. Qu, Y.-M. Shi, and D. Zhao, "Comparative pharmacokinetics of five rhubarb anthraquinones in normal and thrombotic focal cerebral ischemiainduced rats," Phytotherapy Research, vol. 27, no. 10, 2012.

[55] J. S. Kim and L. R. Caplan, "Clinical stroke syndromes," Frontiers of Neurology and Neuroscience, vol. 40, pp. 72-92, 2016.

[56] C. Iadecola, "The neurovascular unit coming of age: a journey through neurovascular coupling in health and disease," Neuron, vol. 96, no. 1, pp. 17-42, 2017.

[57] J. H. Zhang, J. Badaut, J. Tang, A. Obenaus, R. Hartman, and W. J. Pearce, "The vascular neural network-a new paradigm in stroke pathophysiology," Nature Reviews Neurology, vol. 8, no. 12, pp. 711-716, 2012.

[58] L. Zan, X. Zhang, Y. Xi et al., "SRC regulates angiogenic factors and vascular permeability after focal cerebral ischemia-reperfusion," Neuroscience, vol. 262, pp. 118-128, 2014.

[59] T. Hansen, A. Moss, and N. Brindle, "Vascular endothelial growth factor and angiopoietins in neurovascular regeneration and protection following stroke," Current Neurovascular Research, vol. 5, no. 4, pp. 236-245, 2008.

[60] B. R. Stockwell, J. P. Friedmann Angeli, H. Bayir et al., "Ferroptosis: a regulated cell death nexus linking metabolism, redox biology, and disease," Cell, vol. 171, no. 2, pp. 273-285, 2017.

[61] E. Sekerdag, I. Solaroglu, and Y. Gursoy-Ozdemir, "Cell death mechanisms in stroke and novel molecular and cellular treatment options," Current Neuropharmacology, vol. 16, no. 9, pp. 1396-1415, 2018.

[62] J. Anrather and C. Iadecola, "Inflammation and stroke: an overview," Neurotherapeutics, vol. 13, no. 4, pp. 661-670, 2016.

[63] R. Jin, L. Liu, S. Zhang, A. Nanda, and G. Li, "Role of inflammation and its mediators in acute ischemic stroke," Journal of Cardiovascular Translational Research, vol. 6, no. 5, pp. 834-851, 2013.

[64] K. J. Becker, "Inflammation and the silent sequelae of stroke," Neurotherapeutics, vol. 13, no. 4, pp. 801-810, 2016.

[65] Y. Gu, J. Chen, and J. Shen, "Herbal medicines for ischemic stroke: combating inflammation as therapeutic targets," Journal of Neuroimmune Pharmacology, vol. 9, no. 3, pp. 313-339, 2014.

[66] A. Janyou, P. Wicha, J. Jittiwat, A. Suksamrarn, C. Tocharus, and J. Tocharus, "Dihydrocapsaicin attenuates blood brain barrier and cerebral damage in focal cerebral ischemia/ reperfusion via oxidative stress and inflammatory," Scientific Reports, vol. 7, no. 1, p. 10556, 2017.

[67] A. Neves Carvalho, O. Firuzi, M. Joao Gama, J. van Horssen, and L. Saso, "Oxidative stress and antioxidants in neurological diseases: is there still hope?" Current Drug Targets, vol. 18, no. 6, pp. 705-718, 2017.

[68] H. E. de Vries, M. Witte, D. Hondius et al., "Nrf2-induced antioxidant protection: a promising target to counteract ROS-mediated damage in neurodegenerative disease?" Free Radical Biology and Medicine, vol. 45, no. 10, pp. 1375-1383, 2008.

[69] X. Lin, T. Liu, P. Li et al., "iTRAQ-based proteomics analysis reveals the effect of rhubarb in rats with ischemic stroke," BioMed Research International, vol. 2018, Article ID 6920213, 13 pages, 2018.

[70] C. N. Egbujo, D. Sinclair, and C.-G. Hahn, "Dysregulations of synaptic vesicle trafficking in schizophrenia," Current Psychiatry Reports, vol. 18, no. 8, p. 77, 2016.

[71] F. Valtorta, M. Pennuto, D. Bonanomi, and F. Benfenati, "Synaptophysin: leading actor or walk-on role in synaptic vesicle exocytosis?" BioEssays, vol. 26, no. 4, pp. 445-453, 2004.

[72] X. K. Zhao, N. Xiao, J. B. Zhou et al., "Effects of ephedrine on the recovery of motor function in cerebral ischemic rats and the molecular mechanism," Chinese Journal of Rehabilitation Medicine, vol. 20, no. 3, pp. 172-175, 2005.

[73] A. John, E. Ng-Cordell, N. Hanna, D. Brkic, and K. Baker, "The neurodevelopmental spectrum of synaptic vesicle cycling disorders," Journal of Neurochemistry, vol. 157, no. 2, pp. 208-228, 2021. 
[74] A. Margiotta, "Role of SNAREs in neurodegenerative diseases," Cells, vol. 10, no. 5, pp. 991-2021, 2021.

[75] L. I. Benowitz and A. Routtenberg, "GAP-43: an intrinsic determinant of neuronal development and plasticity," Trends in Neurosciences, vol. 20, no. 2, pp. 84-91, 1997.

[76] S. M. Strittmatter, T. Vartanian, and M. C. Fishman, "GAP43 as a plasticity protein in neuronal form and repair," Journal of Neurobiology, vol. 23, no. 5, pp. 507-520, 1992.

[77] R. P. Stroemer, T. A. Kent, and C. E. Hulsebosch, "Enhanced neocortical neural sprouting, synaptogenesis, and behavioral recovery with $\mathrm{d}$-amphetamine therapy after neocortical infarction in rats," Stroke, vol. 29, no. 11, pp. 2381-2395, 1998.

[78] K. Miyake, W. Yamamoto, M. Tadokoro et al., "Alterations in hippocampal GAP-43, BDNF, and L1 following sustained cerebral ischemia," Brain Research, vol. 935, no. 1-2, pp. 24-31, 2002.

[79] Y. Li, N. Jiang, C. Powers, and M. Chopp, "Neuronal damage and plasticity identified by microtubule-associated protein 2 , growth-associated protein 43 , and cyclin D1 immunoreactivity after focal cerebral ischemia in rats," Stroke, vol. 29, no. 9, pp. 1972-1981, 1998.

[80] W. Hua, H. Wu, M. Zhou et al., "Protective effects of recombinant human erythropoietin on oligodendrocyte after cerebral infarction," Zhonghua Bing Li Xue Za Zhi, vol. 44, no. 5, pp. 323-328, 2015, in Chinese.

[81] M. Zuo, H. Guo, T. Wan et al., "Wallerian degeneration in experimental focal cortical ischemia," Brain Research Bulletin, vol. 149, pp. 194-202, 2019.

[82] Y.-Y. Zhao, D.-J. Yan, and Z.-W. Chen, "Role of AIF-1 in the regulation of inflammatory activation and diverse disease processes," Cellular Immunology, vol. 284, no. 1-2, pp. 75-83, 2013.

[83] R. von Bernhardi, F. Heredia, N. Salgado, and P. Muñoz, "Microglia function in the normal brain," Advances in Experimental Medicine and Biology, vol. 949, pp. 67-92, 2016.

[84] N. Gupta, S. Jadhav, K.-L. Tan, G. Saw, K. B. Mallilankaraman, and S. T. Dheen, "miR-142-3p regulates $\mathrm{BDNF}$ expression in activated rodent microglia through its target CAMK2A," Frontiers in Cellular Neuroscience, vol. 14, pp. 132-2020, 2020.

[85] T. Akita, K. Aoto, M. Kato et al., "De novo variants in CAMK2A and CAMK2B cause neurodevelopmental disorders," Annals of Clinical and Translational Neurology, vol. 5, no. 3, pp. 280-296, 2018.

[86] Y. Park and J.-K. Ryu, "Models of synaptotagmin-1 to trigger $\mathrm{Ca}^{2+}$-dependent vesicle fusion," FEBS Letters, vol. 592, no. 21, pp. 3480-3492, 2018.

[87] Z. Xie, J. Long, J. Liu, Z. Chai, X. Kang, and C. Wang, "Molecular mechanisms for the coupling of endocytosis to exocytosis in neurons," Frontiers in Molecular Neuroscience, vol. 10, p. 47, 2017.

[88] B. Wang, L. Pan, M. Wei et al., "FMRP-mediated axonal delivery of miR-181d regulates axon elongation by locally targeting Map1b and Calm1," Cell Reports, vol. 13, no. 12, pp. 2794-2807, 2015.

[89] H. Kobayashi, S. Saragai, A. Naito, K. Ichio, D. Kawauchi, and F. Murakami, "Calm1 signaling pathway is essential for the migration of mouse precerebellar neurons," Development, vol. 142, no. 2, pp. 375-384, 2015.

[90] B. Bae, H. N. Gruner, M. Lynch et al., "Elimination of Calm1 long $3^{\prime}$-UTR mRNA isoform by CRISPR-Cas9 gene editing impairs dorsal root ganglion development and hippocampal neuron activation in mice," $R N A$, vol. 26, no. 10, pp. 1414-1430, 2020.

[91] L. Gu, J. Huang, J. Li et al., "Association of CALM1 rs3179089 polymorphism with ischemic stroke in Chinese Han population," NeuroMolecular Medicine, vol. 20, no. 2, pp. 271-279, 2018.

[92] A. Muller, "Endogenous $\mathrm{Ca}^{2+}$ buffer concentration and $\mathrm{Ca}^{2+}$ microdomains in hippocampal neurons," Journal of Neuroscience, vol. 25, no. 3, pp. 558-565, 2005.

[93] Q. Guo, S. Christakos, N. Robinson, and M. P. Mattson, "Calbindin D28k blocks the proapoptotic actions of mutant presenilin 1: reduced oxidative stress and preserved mitochondrial function," Proceedings of the National Academy of Sciences, vol. 95, no. 6, pp. 3227-3232, 1998.

[94] B. Schwaller, "Cytosolic Ca ${ }^{2+}$ buffers," Cold Spring Harbor Perspectives in Biology, vol. 2, no. 11, Article ID a004051, 2010.

[95] B. Schwaller, "The use of transgenic mouse models to reveal the functions of $\mathrm{Ca}^{2+}$ buffer proteins in excitable cells," Biochimica et Biophysica Acta (BBA)-General Subjects, vol. 1820, no. 8, pp. 1294-1303, 2012.

[96] M. Zhao and A. T. Brunger, "Recent advances in deciphering the structure and molecular mechanism of the AAA+ ATPase N-Ethylmaleimide-Sensitive factor (NSF)," Journal of Molecular Biology, vol. 428, no. 9, pp. 1912-1926, 2016.

[97] T. C. Südhof and J. Rizo, "Synaptic vesicle exocytosis," Cold Spring Harbor Perspectives in Biology, vol. 3, no. 12, Article ID a005637, 2011.

[98] X. Lou, "Sensing exocytosis and triggering endocytosis at synapses: synaptic vesicle exocytosis-endocytosis coupling," Frontiers in Cellular Neuroscience, vol. 12, p. 66, 2018.

[99] T. Ziemssen, K. Akgün, and W. Brück, "Molecular biomarkers in multiple sclerosis," Journal of Neuroinflammation, vol. 16, no. 1, p. 272, 2019.

[100] T. M. Evans, H. V. Remmen, A. Purkar et al., "Microwave and magnetic (M2) proteomics of a mouse model of mild traumatic brain injury," Translational Proteomics, vol. 3, pp. 10-21, 2014.

[101] M. Gunnarsson, C. Malmeström, M. Axelsson et al., “Axonal damage in relapsing multiple sclerosis is markedly reduced by natalizumab," Annals of Neurology, vol. 69, no. 1, pp. 83-89, 2011.

[102] M. Cao, Y. Fang, W. Jia, Y. Wang, J. Sun, and D. Tao, "Emodin relieves hypoxia-triggered injury via elevation of microRNA-25 in PC-12 cells," Artificial Cells, Nanomedicine, and Biotechnology, vol. 47, no. 1, pp. 2678-2687, 2019.

[103] J. Liu, F. Kuang, G. Kroemer, D. J. Klionsky, R. Kang, and D. Tang, "Autophagy-dependent ferroptosis: machinery and regulation," Cell Chemical Biology, vol. 27, no. 4, pp. 420-435, 2020.

[104] Z. Peng, J. Li, Y. Li et al., "Downregulation of miR-181b in mouse brain following ischemic stroke induces neuroprotection against ischemic injury through targeting heat shock protein A5 and ubiquitin carboxyl-terminal hydrolase isozyme L1," Journal of Neuroscience Research, vol. 91, no. 10, pp. 1349-1362, 2013.

[105] Z. F. Peng, J. Meng, and J. H. Zhang, "The role of heat shock protein A5-mediated autophagy in cerebral ischemia/ reperfusion injury in mice," Chinese Journal of Applied Physiology, vol. 33, no. 3, pp. 234-238, 2017.

[106] C. F. Peng, "Role of microRNA-181b in ischemic stroke mice through regulation of heat shock protein A5," Journal of Anatomy, vol. 48, no. 1, pp. 25-29, 2017. 
[107] S. Liddelow and D. Hoyer, "Astrocytes: adhesion molecules and immunomodulation," Current Drug Targets, vol. 17, no. 16, pp. 1871-1881, 2016.

[108] M. Winther, V. Berezin, and P. S. Walmod, "NCAM2/ OCAM/RNCAM: cell adhesion molecule with a role in neuronal compartmentalization," The International Journal of Biochemistry \& Cell Biology, vol. 44, no. 3, pp. 441-446, 2012.

[109] M. Mehrabian, H. Hildebrandt, and G. Schmitt-Ulms, "NCAM1 polysialylation," ASN Neuro, vol. 8, no. 6, 2016.

[110] V. Berezin, P. S. Walmod, M. Filippov, and A. Dityatev, "Targeting of ECM molecules and their metabolizing enzymes and receptors for the treatment of CNS diseases," Progress in Brain Research, vol. 214, pp. 353-388, 2014.

[111] O. Reiner, "LIS1 and DCX: implications for brain development and human disease in relation to microtubules," Scientific, vol. 2013, Article ID 393975, 17 pages, 2013.

[112] O. Reiner, A. Gdalyahu, I. Ghosh et al., "DCX's phosphorylation by not just another kinase (JNK)," Cell Cycle (Georgetown, Tex.), vol. 3, no. 6, pp. 747-751, 2004.

[113] F. Wang, Neural Differentiation of Hippocampal Dentate Gyrus after Ischemic Stroke in Youth and the Role and Mechanism of Paclitaxel Intervention in Ischemic Stroke, Yangzhou University, Yangzhou, China, 2021.

[114] X. Zhu, Z. Long, T. Bao, L. Liu, and K. Yang, "Exploring the mechanism of radix rhei et rhizome intervention in intracerebral hemorrhage based on systematic pharmacology and proteomics strategy," Bioscience Reports, vol. 41, no. 3, Article ID BSR20201910, 2021. 